\title{
EXCEPTIONAL VALUES OF MEROMORPHIC FUNCTIONS
}

\author{
BY \\ E. F. COLLINGWOOD
}

1. Introduction. We consider a function $f(z)$ which is meromorphic and not rational in the domain $|z|<R \leqq \infty$. Let $a$ be any complex number, infinity not excluded. A zero of $f(z)-a$, when $a \neq \infty$, or a pole of $f(z)$ when $a=\infty$, will be called an $a$-point of $f(z)$. In the standard notation( $\left.{ }^{1}\right) n(r, a)$ $=n(r, 1 /(f-a))$ is the number of $a$-points of $f(z)$ within and on the circumference $|z|=r$, multiple $a$-points being counted with their order of multiplicity. Further

$$
\begin{aligned}
& N(r, a)=N\left(r, \frac{1}{f-a}\right)=\int_{0}^{r} \frac{n(t, a)-n(0, a)}{t} d t+n(0, a) \log r ; \\
& N(r, \infty)=N(r, f) .
\end{aligned}
$$

We adopt the plane as opposed to the spherical definition of $m(r, a)$, namely

$$
m(r, a)=m\left(r, \frac{1}{f-a}\right)=\frac{1}{2 \pi} \int_{0}^{2 \pi} \log ^{+}\left|\frac{1}{f\left(r e^{i \phi}\right)-a}\right| d \phi
$$

and

$$
m(r, \infty)=m(r, f)=\frac{1}{2 \pi} \int_{0}^{2 \pi} \log ^{+}\left|f\left(r e^{i \phi}\right)\right| d \phi .
$$

Then

$$
T(r)=T(r, f)=m(r, f)+N(r, f)
$$

is Nevanlinna's characteristic for the function $f(z)$ and, for any complex $a$,

$$
T(r)=m(r, a)+N(r, a)+O(1) .
$$

The deficiency $\delta(a)$ of the value $a$ is defined by the equation

$$
\delta(a)=\liminf _{r=R} \frac{m(r, a)}{T(r)}=1-\limsup _{r=R} \frac{N(r, a)}{T(r)} .
$$

Plainly $0 \leqq \delta(a) \leqq 1$. A value $a$ is said to be deficient if $\delta(a)>0$.

Presented to the Society, September 10, 1948; received by the editors April 23, 1948.

(1) The general reference for the theory is to R. Nevanlinna's two standard books: (i) Théorème de Picard-Borel et la Théorie des Fonctions Méromorphes, Gauthier-Villars, 1929, cited hereafter as F.M.; (ii) Eindeutige Analytische Funktionen, Springer, 1936, cited as E.A.F. 
The upper (or Valiron) $\left({ }^{2}\right)$ deficiency of the value $a$ is defined by

$$
\Delta(a)=\lim _{r=R} \sup \frac{m(r, a)}{T(r)}=1-\liminf _{r=R} \frac{N(r, a)}{T(r)}
$$

from which it follows that

$$
0 \leqq \delta(a) \leqq \Delta(a) \leqq 1 .
$$

For any function $f(z)$ having $T(r)=T(r, f)$ unbounded, deficiency of either type is an exceptional property $\left({ }^{3}\right)$. For such a function, and we shall confine ourselves exclusively to such functions, the distribution of $a$-points is anomalous if $\Delta(a)>0$; and a fortiori if $\delta(a)>0$. A value $a$ for which $\Delta(a)>0$ we call an exceptional value and a value for which $\Delta(a)=0$ a normal value $\left(^{4}\right)$ of $f(z)$.

The study of exceptional values may be pursued from either of two points of view. We may study a whole category of exceptional values, for example the set of deficient values, or we may fix our attention upon an individual exceptional value and investigate its properties. The purpose of this paper is to make a contribution to the study of individual exceptional values. Theorems of a limited character bearing on this problem have been known from the inception of the Nevanlinna theory. These have taken the form of sufficiency conditions for $\Delta(a)=0$ with, as corollaries, necessary conditions for $\Delta(a)>0$.

Denote by $S$ the Riemann surface on which the function $w=f(z)$ maps its domain of existence $|z|<R \leqq \infty$ and let $\sigma$ be a positive number. The primitive result is the "schlicht condition" theorem of the writer $\left(^{5}\right)$. The sharpest form of this theorem is the following due to Teichmüller $\left(^{6}\right)$.

If over the domain $|w-a|<\sigma$ (or $1 /|w|<\sigma$ if $a=\infty$ ) the surface $S$ has only schlicht sheets all of whose boundary points are internal points of $S$, then

$$
m(r, a)=O(1)
$$

and hence, if $T(r)$ is unbounded, $\Delta(a)=0$.

The proof of this theorem, like the proofs of its forerunners, depends upon an application of Koebe's distortion theorem to the inverse $z=z(w)$ of $f(z)$

(2) Valiron [1]. The terminology is that of Ullrich [4, p. 192]. For consistency $\delta(a)$ might be called the lower or Nevanlinna deficiency. But this is an unnecessary complication. The numbers in brackets refer to the bibliography at the end.

(3) See E.A.F. pp. 254-264 or F.M. chap. 6.

(4) Nevanlinna uses normal in the sense of not deficient; but the sense proposed here seems the more appropriate. [1].

(5) Collingwood [1], Cartan [1], Selberg [1] and [2]. For a related theorem see Dinghas

(6) Teichmüller [1, pp. 99-103]. This paper contains a bibliography of the problem up to 1936. As this is not very easily accessible I have incorporated it, irrespective of specific reference to the items, in the bibliography at the end. 
and the theorem has been habitually stated in this "inverse" form. But a direct form of the theorem is perhaps more immediately suggestive of its bearing upon the distribution of $a$-points. For this we require a preliminary definition.

For $a \neq \infty$ the inequality $|f(z)-a|<\sigma$ is satisfied in a set of distinct connected domains whose frontiers within the domain $|z|<R \leqq \infty$ consist of level curves on which $|f(z)-a|=\sigma$. A typical domain of this (possibly infinite) set of domains is denoted by $G(a, \sigma)$. For $a=\infty$ we denote a typical domain in which $|1 / f(z)|<\sigma$ by $G(\infty, \sigma)\left({ }^{7}\right)$. We say that $G(a, \sigma)$ is "bounded" if its closure $\bar{G}(a, \sigma)\left({ }^{8}\right)$ is contained in the domain $|z|<R \leqq \infty$; otherwise $G(a, \sigma)$ is "unbounded" $\left({ }^{9}\right)$.

A "bounded" $G(a, \sigma)$ in which $f(z)$ is schlicht is mapped by $f(z)$ on a schlicht sheet, covering $|w-a|<\sigma$ (or $|1 / w|<\sigma$ if $a=\infty$ ), all of whose frontier points are internal points of $S$. We may accordingly put the schlichtcondition theorem in the following direct form.

If, for $a$ given complex $a$ and $\sigma>0, f(z)$ is schlicht in every domain $G(a, \sigma)$ and if none of these domains is "unbounded," then (1.1) is satisfied.

Under the condition of the theorem each domain $G(a, \sigma)$ is bounded by a single closed level curve on which $|f(z)-a|=\sigma$ (or $|1 / f(z)|=\sigma$ if $a=\infty$ ) and contains a single $a$-point of $f(z)$. In an obvious sense the $a$-points are isolated by the closed level curves and this is a sufficient condition for their distribution to be normal provided that $T(r)$ is unbounded.

For a long time attempts to generalize this theorem by relaxing the requirement that $f(z)$ shall be schlicht in the domains $G(a, \sigma)$ were unsuccessful. But recently the difficulty has been overcome by H. L. Selberg $\left({ }^{10}\right)$ who has proved the following theorem.

If over the domain $|w-a|<\sigma$ (or $|1 / w|<\sigma$ if $a=\infty$ ) the surface $S$ consists of portions of not more than $p$ sheets all of whose frontier points are internal points of $S$, then

$$
m(r, a)<p \log r+\log ^{+} \frac{1}{\sigma}+O(1)
$$

(7) This notation is in general adequate, but where it is necessary to indicate the relative function we may use the full notation $G(a, \sigma)=G(1 /(f-a), \sigma) ; G(\infty, \sigma)=G(f, \sigma)$.

(8) We use the notation of Newman, Topology of plane sets, Cambridge, 1939.

( ${ }^{9}$ If the "diameter" of $G(a, \sigma)$ is defined as the upper bound of the distance between pairs of points of $G(a, \sigma)$ in terms of the Euclidean metric if $R=\infty$ and in terms of the hyperbolic metric if $R<\infty, G(a, \sigma)$ is "bounded" or "unbounded" according as its diameter is finite or infinite.

(10) Selberg [3] and [4]. The theorem was stated without proof substantially in the above form by Ullrich [2] and in a modified form, with a constant in place of $\log r$, by Mme. LaurentSchwartz [1]. As was pointed out by Teichmüller $[1$, footnote 8$]$, the argument by which Tumura [1] sought to prove a similar theorem is inadequate. 
It follows that if $f(z)$ is non-rational and if, for $R<\infty, T(r)$ is unbounded, then $\Delta(a)=0$.

The corresponding direct theorem is the following.

If, for a given complex $a$ and $\sigma>0, f(z)$ is of valency ${ }^{11}{ }^{11}$ not greater than $p$ in any of the domains $G(a, \sigma)$ and if none of these domains is "unbounded," then (1.2) is satisfied.

For $p>1$ the domains $G(a, \sigma)$ may be multiply-connected and the conformal mapping methods that suffice in the schlicht case are no longer applicable. Selberg's decisive step was to reduce the problem to one of potential theory which can be solved for multiply-connected domains.

Under the conditions of Selberg's theorem the $a$-points of $f(z)$ are segregated in groups of not more than $p$ members within the bounded domains $G(a, \sigma)$ and this is again a sufficient condition for their distribution to be normal if $f(z)$ is non-rational and has $T(r)$ unbounded.

As corollaries of the foregoing theorems we can derive necessary conditions for $\Delta(a)>0$ and, a fortiori, for $\delta(a)>0$. But these conditions are qualitative and non-metrical in the sense that they are unrelated to the value of $\Delta(a)$ or $\delta(a)$. By formulating appropriate definitions which enable us to replace $\sigma$ and $p$ in the inequalities (1.1) and (1.2) by functions of $r$, which we shall designate by $\sigma(r)$ and $p(r)$, or to establish analogous inequalities with these functions we can, however, obtain metrical results; it is this that we set out to do in a systematic way in the present paper.

The main theorem that we shall prove, namely Theorem II, generalizes the theorems of Selberg and Teichmüller in the way described and is proved by their methods which are applicable with only minor adaptations to the generalized conditions. The essential idea of our method is in fact introduced with the definitions; and the formal pattern of theorems and associated groups of corollaries which they impose provides the framework for the systematic derivation of metrical relations from Theorem II. The introduction of these definitions leads first, however, to a restatement of the known results in a rather more general form in accordance with this pattern; and these are included for completeness.

The paper $\left.{ }^{12}\right)$ is in four parts. I ( $\left.\$ 2-9\right)$ is concerned with the definitions and preparatory results arising from them. In II ( $\$ \$ 10-13)$ these definitions are applied, as part of the systematic treatment of the subject, to the generalized restatement and discussion of the known results. The theorems and corollaries in this part, which are based upon a theorem that is new in

(11) The definition of valency is that of Montel. $f(z)$ is of valency $p$ in a domain $D$ if it takes no value more than $p$ times and at least one value $p$ times in $D$. Montel $[1$, p. 4$]$.

(12) Note added in proof: A summary of the results of this paper appeared in a series of notes in C.R. Acad. Sci. Paris: Collingwood $[3 ; 4 ; 5 ; 6]$. The same ideas have since been applied by Parreau [1] to generalize a schlicht condition theorem for Ahlfors deficiency due to Dinghas [1]. 
form rather than in content, are designated by capital letters. III (\$\$14-22) contains the systematic development of the new results from the main theorem. The theorems and corollaries in this part are designated by Roman numerals. To avoid a long digression in III the proof of the main theorem is given separately in IV ( $\$ 23-25)$.

\section{Definitions AND PRELIMINARIES}

2. The surface $S$. We begin by recalling briefly the relevant properties of the Riemann surface $S$ on which the meromorphic function $w=f(z)$ maps its domain of existence $|z|<R \leqq \infty\left({ }^{13}\right) . S$ may be taken either as spread over the $w$-plane or the $w$-sphere. We shall take it as spread over the plane. Since $f(z)$ is non-rational $S$ has an infinity of sheets. The surface $S$ is of parabolic or hyperbolic type according as $R=\infty$ or $R<\infty$.

The inverse function $z=z(w)$ of $w=f(z)$ is uniform on the surface $S$ which it maps on the domain $|z|<R \leqq \infty$. The winding points of $S$ of finite order correspond to algebraic elements of $z(w)$ and are designated algebraic points. They are internal points of $S$. The boundary places of $S$ are winding points of infinite order and correspond to transcendant critical points of $z(w)$ and to the asymptotic values of $f(z)$. A number $\omega$ is an asymptotic value of $f(z)$ if there is a continuous Jordan curve $\Gamma$ in $|z|<R \leqq \infty$ tending to the frontier of this domain on which $f(z) \rightarrow \omega$ as $|z| \rightarrow R \leqq \infty$. In the parabolic case $\Gamma$ tends to the unique frontier point of the domain of existence of $f(z)$, which we have taken to be the point $z=\infty$. But in the hyperbolic case the properties of $\Gamma$ can be conspicuously different according as $T(r)$ is bounded or unbounded. The frontier of the domain of existence of $f(z)$ is now the whole circumference $|z|=R$ and the limiting set of $\Gamma$ on this circumference may be either a single point or a whole arc. The latter case cannot arise if $T(r)$ is bounded. For then if $\Gamma$ tends to an arc of $|z|=R$ the radial limit of $f(z)$, which exists for almost all points of this arc, cannot differ from $\omega$ at any point of the arc and it follows from a well known theorem that $f(z) \equiv \omega$. In the contrary sense Valiron [2] has constructed a function which is regular and of infinite order in $|z|<1$ and has an arbitrary number of distinct spiral asymptotic paths $\Gamma$ converging to the circumference $|z|=1$. This function of course has no radial limits anywhere on $|z|=1$. An example for which $T(r)$ is unbounded and $f(z)$ has a radial limit at a set of points of $|z|=1$ which is everywhere dense is furnished by the modular function. In this case there can be no asymptotic path $\Gamma$ tending to an arc of $|z|=1$. These examples illustrate significant points of difference that we must keep in view but by no means cover the full range of possibilities.

The curve $\Gamma$ is mapped by $f(z)$ on a curve in $S$ tending to a boundary

${ }^{(13)}$ See E.A.F. pp. 269-277 for a general account. A detailed study of the parabolic case is contained in Iversen's well known Thése: Iversen [1]. For expository accounts of more recent developments see Ullrich [3] and [4]. 
place of $S$ over $w=\omega$ and the corresponding branch of $z(w)$ has a transcendant point at $w=\omega$.

3. The domains $U(a, \sigma)$ and $G(a, \sigma) . T(r)$ is unbounded in the parabolic case $R=\infty$. We shall assume throughout that $T(r)$ is also unbounded in the hyperbolic case $R<\infty$; or, in other words, that $f(z)$ is not of bounded characteristic. The $\sigma$-neighbourhood of any point $a$ in the complex $w$-plane, defined by the inequality $|w-a|<\sigma$ (or $|1 / w|<\sigma$ if $a=\infty$ ) is then covered by a finite or infinite set of distinct connected portions of $S$. These are Riemannian domains. A typical domain of the set will be denoted by $U(a, \sigma)=U(1 /(f-a), \sigma$ ) (or $U(f, \sigma)$ if $a=\infty)$. We may note explicitly that a $U(a, \sigma)$ having more than one winding point may be multiply connected.

To avoid repetition all subsequent definitions involving $a$ will be stated in the form for $a \neq \infty$, it being understood that the corresponding form for $a=\infty$ is obtained on writing $1 / w$ for $w-a$ or $1 / f(z)$ for $f(z)-a$. Except where it is specifically stated to the contrary all our theorems are valid for finite or infinite values of $a$.

Each $U(a, \sigma)$ is mapped by $z(w)$ on a domain $G(a, \sigma)$ as defined in $\S 1$. A domain $U(a, \sigma)$ is said to be bounded or unbounded $\left({ }^{14}\right)$ according as the corresponding domain $G(a, \sigma)$ on which it is mapped is bounded or unbounded. The frontier of a bounded $G(a, \sigma)$ consists of a finite number of closed level curves on which $|f(z)-a|=\sigma$ and, for all $\alpha$ in $|\alpha-a|<\sigma$, it contains the same finite number, say $p$, of $\alpha$-points of $f(z)$, multiple points being counted with their order of multiplicity. $p$ is the valency of $f(z)$ in $G(a, \sigma)$. The corresponding bounded $U(a, \sigma)$ is a Riemannian domain of $p$ sheets each covering the domain $|w-a|<\sigma$ and joined at a finite number of algebraic points. The frontier of this $U(a, \sigma)$ consists of a finite set of closed circuits over the circumference $|w-a|=\sigma$, and every point of these circuits is an internal point of $S$. A bounded $U(a, \sigma)$ thus has a relatively simple structure, although it may be of arbitrarily high connectivity.

Now consider an unbounded domain $G(a, \sigma)$ and suppose that for all $\alpha$ in $|\alpha-a|<\sigma$ the number of $\alpha$-points of $f(z)$ in $G(a, \sigma)$ does not exceed $K<\infty$. If $p$ is the smallest integral value of $K$ for which this condition is satisfied, then $f(z)$ is of valency $p$ (or $p$-valent) in $G(a, \sigma)$ and the unbounded Riemannian domain $U(a, \sigma)$ on which $f(z)$ maps $G(a, \sigma)$ has $p$ sheets. If there is no finite $K$ for which the condition is satisfied, $f(z)$ is of infinite valency in $G(a, \sigma)$, the corresponding $U(a, \sigma)$ has an infinity of sheets, and we write $p=\infty$.

4. The domains $U(r, a, \sigma)$ and $G(r, a, \sigma)$. The circular domain $|z|<r$ $<R \leqq \infty$ is mapped by $f(z)$ on a surface $S_{r}$ which is a simply-connected portion of $S$ having a finite number of sheets. The frontier of $S_{r}$ is a closed curve $C_{r}$ corresponding to the circumference $|z|=r$. Every point of $C_{r}$ is an

(14) The quotation marks will now be omitted whenever the sense in which the terms bounded and unbounded are being used is clear from the context. 
internal point of $S$. As $r \rightarrow R$ the surface $S_{r}$ exhausts $S$.

Every domain $G(a, \sigma)$ satisfies one of the following three conditions:

(i) Every point of $G(a, \sigma)$ is a point of the domain $|z|<r$.

(ii) No point of $G(a, \sigma)$ is a point of the domain $|z|<r$.

(iii) $G(a, \sigma)$ contains points of the circumference $|z|=r$.

Condition (i) is excluded if $G(a, \sigma)$ is unbounded. Corresponding alternatives for $U(a, \sigma)$ are stated in terms of $S_{r}$ and $C_{r}$.

A typical domain $G(a, \sigma)$ which satisfies condition (iii) is denoted by $G(r, a, \sigma)=G(r, 1 /(f-a), \sigma)$ and the corresponding $U(a, \sigma)$, on which it is mapped by $f(z)$, by $U(r, a, \sigma)=U(r, 1 /(f-a), \sigma)$.

Since $f(z)$ has no singularities other than poles in $|z|<R$ the number of the domains $G(r, a, \sigma)$ (and $U(r, a, \sigma)$ ) for a given $r$ is finite and will be denoted by $\lambda(r)$.

Where necessary to avoid ambiguity an individual $G(r, a, \sigma)$ or $U(r, a, \sigma)$ will be distinguished by a suffix $\nu$ as $G_{\nu}(r, a, \sigma)$ or $U_{\nu}(r, a, \sigma)$, where $1 \leqq \nu \leqq \lambda(r)$.

5. The domains $U(r, a, \sigma(r))$ and $G(r, a, \sigma(r))$. The definitions of $\S 4$ remain applicable when $\sigma$ is replaced by a single-valued function $\sigma(r)$ defined in the semi-open interval $0 \leqq r<R \leqq \infty$ in which it has only isolated ordinary discontinuities. For a given value of $r$ the $G(a, \sigma(r))$ are the domains in which $|f(z)-a|<\sigma(r)$ and those of them, if any, which contain points of the circumference $|z|=r$ are denoted generally by $G(r, a, \sigma(r))=G(r, 1 /(f-a), \sigma(r))$. The number of the domains $G(r, a, \sigma(r))$ for a given $r$ is finite and is again denoted by $\lambda(r)$. An individual $G(r, a, \sigma(r))$ is distinguished by a suffix $\nu$ as $G_{\nu}(r, a, \sigma(r))$.

The corresponding Riemannian domains are denoted by $U(r, a, \sigma(r))$ $=U(r, 1 /(f-a), \sigma(r))$ or $U_{\nu}(r, a, \sigma(r))=U_{\nu}(r, 1 /(f-a), \sigma(r))$.

6. $\sigma$ and $\sigma(r)$ as levels relative to $f(z)$ and $a$. We now introduce the conception of a level. For a given value of $r$ the parts of the frontiers of the domains $G(r, a, \sigma)$, if any such domains exist, within the domain $|z|<R \leqq \infty$ consist of level curves on which $|f(z)-a|=\sigma$. We shall then say that $\sigma$ is a level of positive valency relative to $f(z)$ and a for this value of $r$. If no domain $G(r, a, \sigma)$ exists, that is, if $\left|f\left(r e^{i \phi}\right)-a\right| \geqq \sigma$ for all $0 \leqq \phi<2 \pi$, we say that $\sigma$ is a null level relative to $f(z)$ and a for this value of $r$.

If all the domains $G(r, a, \sigma)$ are bounded for a given value of $r$, then their frontiers consist of a finite set of closed level curves contained in $|z|<R \leqq \infty$ and we say that $\sigma$ is a closed level relative to $f(z)$ and a for this value of $r$. If at least one of the $G(r, a, \sigma)$ is unbounded $\sigma$ is an open level relative to $f(z)$ and $a$ for this value of $r$.

The above definitions apply without modification when $\sigma$ is replaced by a single-valued function $\sigma(r)$ defined in the semi-open interval $0 \leqq r<R \leqq \infty$ in which it has only isolated ordinary discontinuities. We shall assume $\sigma(r)$ to be subject to this condition throughout. For a given $r, \sigma(r)$ is a null level relative to $f(z)$ and $a$ if $\left|f\left(r e^{i \phi}\right)-a\right| \geqq \sigma(r)$ for all $0 \leqq \phi<2 \pi$. A level $\sigma(r)$ which 
is not null relative to $f(z)$ and $a$ for a given $r$ is of positive valency, closed if all the $G(r, a, \sigma(r))$ are bounded and otherwise open.

The definitions may also be formulated in "inverse" language, $U(r, a, \sigma)$ and $U(r, a, \sigma(r))$ taking the place of $G(r, a, \sigma)$ and $G(r, a, \sigma(r))$.

7. The valencies $P(r, a, \sigma)$ and $P(r, a, \sigma(r))$. With the concept of a level we associate a valency.

For a given value of $r$ the valency $P(r, a, \sigma)=P(r, 1 /(f-a), \sigma)$ of a level $\sigma$ relative to $f(z)$ and $a$ is, by definition, the maximum value of the valency of $f(z)$ in any one of the domains $G(r, a, \sigma)$. Thus, denoting by $p_{\nu}(r, a, \sigma)$ the valency of $f(z)$ in a domain $G_{\nu}(r, a, \sigma)$,

$$
P(r, a, \sigma)=\max _{\nu \leqq \lambda(r)} p_{\nu}(r, a, \sigma) .
$$

For $a=\infty$ we work with $P(r, \infty, \sigma)=P(r, f, \sigma)$. Clearly, if $\sigma_{1}<\sigma$, then

$$
P\left(r, a, \sigma_{1}\right) \leqq P(r, a, \sigma) .
$$

For every domain $G\left(r, a, \sigma_{1}\right)$ is contained in a domain $G(r, a, \sigma)$.

Generalizing the above, the valency of the level $\sigma(r)$ relative to $f(z)$ and $a$ is, by definition,

$$
P(r, a, \sigma(r))=\max _{\nu \leqq \lambda(r)} p_{\nu}(r, a, \sigma(r)),
$$

where $p_{\nu}(r, a, \sigma(r))$ is the valency of $f(z)$ in $G_{\nu}(r, a, \sigma(r))$, with a corresponding definition for $a=\infty$. For a function $\sigma_{1}(r) \leqq \sigma(r)$ we have, as above,

$$
P\left(r, a, \sigma_{1}(r)\right) \leqq P(r, a, \sigma(r)) .
$$

If $\sigma(r)$ is a null level relative to $f(z)$ and $a$, we put $P(r, a, \sigma(r))=0$. If $\sigma(r)$ is a closed level relative to $f(z)$ and $a$, then evidently $P(r, a, \sigma(r))<\infty$. If $\sigma(r)$ is an open level relative to $f(z)$ and $a$, then $P(r, a, \sigma(r)) \leqq \infty ;$ and $P(r, a, \sigma(r))=\infty$ if there is at least one unbounded domain $G_{\nu}(r, a, \sigma(r))$ with $p_{\nu}(r, a, \sigma(r))=\infty$. We shall adopt the following terminology. If for a given $r, P(r, a, \sigma(r))=1$ we say that $\sigma(r)$ is a (closed or open) schlicht level relative to $f(z)$ and $a$ for this value of $r$; and if $P(r, a, \sigma(r))=p$, where $1 \leqq p \leqq \infty$, we say that $\sigma(r)$ is a (closed or open) level of valency $p$ relative to $f(z)$ and $a$ for this value of $r$.

Since $p_{\nu}(r, a, \sigma(r))$ is the number of sheets in $U_{\nu}(r, a, \sigma(r))$ these definitions may also be formulated in "inverse" terms.

8. The sets determined by properties of a level relative to $f(z)$ and $a$. For a given constant $\sigma>0$ or a given function $\sigma(r)>0$ the properties of being a null level, or a closed or open level relative to $f(z)$ and $a$ with or without restriction on valency, determine sets of values of $r$ in the interval $0 \leqq r<R \leqq \infty$. The sets with which we shall be concerned are defined in this section.

$V(a, \sigma, 0)=V(1 /(f-a), \sigma, 0)$ is the set of values of $r$ in $0 \leqq r<R \leqq \infty$ for which $\sigma$ is a null level relative to $f(z)$ and $a . V(a, \sigma, p)=V(1 /(f-a), \sigma, p)$, 
$0 \leqq p<\infty$, is the set for which $\sigma$ is either a closed or open level relative to $f(z)$ and $a$ of valency $P(r, a, \sigma) \leqq p$.

From this definition it follows that

$$
V(a, \sigma, p)=\sum_{q=0}^{p} V(a, \sigma, q) .
$$

The set for which $\sigma$ is either a closed or open level relative to $f(z)$ and $a$ of finite (but not necessarily bounded) valency is defined by

$$
\mathfrak{B}(a, \sigma)=\mathfrak{B}\left(\frac{1}{f-a}, \sigma\right)=\sum_{q=0}^{\infty} V(a, \sigma, q) .
$$

The complements of these sets with respect to the interval $0 \leqq r<R$ are denoted by $C V(a, \sigma, 0), C V(a, \sigma, p)$ and $C \mathfrak{B}(a, \sigma)$ respectively. Then

$$
C V(a, \sigma, p)=\prod_{q=0}^{p} C V(a, \sigma, q)
$$

and

$$
C \mathfrak{B}(a, \sigma)=\prod_{q=0}^{\infty} C V(a, \sigma, q) .
$$

$E(a, \sigma, p)=E(1 /(f-a), \sigma, p), 0 \leqq p<\infty$, is the set in which $\sigma$ is a closed level relative to $f(z)$ and $a$ of valency $P(r, a, \sigma) \leqq p$, subject to the convention

$$
E(a, \sigma, 0)=V(a, \sigma, 0) \text {. }
$$

It follows that

$$
E(a, \sigma, p)=\sum_{q=0}^{p} E(a, \sigma, q) .
$$

The set for which $\sigma$ is a closed level relative to $f(z)$ and $a$, necessarily of finite valency, is defined by

$$
\mathfrak{E}(a, \sigma)=\mathfrak{E}\left(\frac{1}{f-a}, \sigma\right)=\sum_{q=0}^{\infty} E(a, \sigma, q) .
$$

The complements of these sets with respect to $0 \leqq r<R$ are denoted by $C E(a, \sigma, p)$ and $C(\xi(a, \sigma)$, where

$$
\begin{aligned}
C E(a, \sigma, p) & =\prod_{q=0}^{p} C E(a, \sigma, q), \\
C \&(a, \sigma) & =\prod_{q=0}^{\infty} C E(a, \sigma, q) .
\end{aligned}
$$


The above definitions hold without modification when $\sigma$ is replaced by a single-valued function $\sigma(r)$. Thus $E(a, \sigma(r), 0)=V(a, \sigma(r), 0)$ is the set in which $\sigma(r)$ is a null level relative to $f(z)$ and $a$.

$$
\mathfrak{B}(a, \sigma(r))=\sum_{q=0}^{\infty} V(a, \sigma(r), q)
$$

is the set in which $\sigma(r)$ is a level of valency $P(r, a, \sigma(r))<\infty$; and

$$
\leftleftarrows(a, \sigma(r))=\sum_{q=0}^{\infty} E(a, \sigma(r), q)
$$

is the set in which $\sigma(r)$ is a closed level relative to $f(z)$ and $a$.

Generalizing a stage further, let $p(r)$ be single-valued, satisfy $0 \leqq p(r)<\infty$ and have only isolated ordinary discontinuities in $0 \leqq r<R$. We then define $V(a, \sigma(r), p(r))$, as the set of values of $r$ in $0 \leqq r<R$ in which $\sigma(r)$ is a closed or open level relative to $f(z)$ and $a$ of valency $P(r, a, \sigma(r)) \leqq p(r)$ and $E(a, \sigma(r), p(r))$ as the set in which $\sigma(r)$ is a closed level relative to $f(z)$ and $a$ of valency $P(r, a, \sigma(r)) \leqq p(r) . C V(a, \sigma(r), p(r))$ and $C E(a, \sigma(r), p(r))$ are the complements of these sets.

By the definitions we have

$$
E(a, \sigma, p) \subseteq V(a, \sigma, p)
$$

whence

$$
\mathfrak{S}(a, \sigma) \subseteq \mathfrak{B}(a, \sigma) .
$$

(8.2) Plainly, if $r_{0}<r_{1}<R$ and $r_{0} \in C \mathfrak{B}(a, \sigma)$, then $r_{1} \in C \mathfrak{B}(a, \sigma)$. Hence $C \mathfrak{B}(a, \sigma)$ is void if $R$ is a limit point of $\mathfrak{B}(a, \sigma)$.

(8.21) Similarly, if $r_{0} \in C \&(a, \sigma)$, then $r_{1} \in C \&(a, \sigma)$ and so $C \&(a, \sigma)$ is void if $R$ is a limit point of $\mathbb{E}(a, \sigma)$.

Also, by the definitions, if $0 \leqq p<p_{1}<\infty$,

$$
V(a, \sigma, p) \subseteq V\left(a, \sigma, p_{1}\right) \subseteq \mathfrak{B}(a, \sigma)
$$

and

$$
E(a, \sigma, p) \subseteq E\left(a, \sigma, p_{1}\right) \subseteq \&(a, \sigma) .
$$

(8.4) It follows from (8.2) and (8.3) that if, for any $p<\infty, R \leqq \infty$ is a limit point of $V(a, \sigma, p)$, then $R$ is also a limit point of $\mathfrak{B}(a, \sigma)$ and so $C \mathfrak{B}(a, \sigma)$ is void.

(8.41) Similarly, from (8.21) and (8.31), if $R$ is a limit point of $E(a, \sigma, p)$, then $C \&(a, \sigma)$ is void. and so

If $0<\sigma_{1}<\sigma$ and $0 \leqq p<p_{1}<\infty$, then $r \in V(a, \sigma, p)$ implies $r \in V\left(a, \sigma_{1}, p\right)$

$$
V(a, \sigma, p) \subseteq V\left(a, \sigma_{1}, p\right) \subseteq V\left(a, \sigma_{1}, p_{1}\right)
$$


Similarly,

$$
E(a, \sigma, p) \subseteq E\left(a, \sigma_{1}, p\right) \subseteq E\left(a, \sigma_{1}, p_{1}\right) .
$$

The corresponding relations when $\sigma$ and $p$ are replaced by $\sigma(r)$ and $p(r)$, where $0<\sigma(r)<\infty$ and $0 \leqq p(r)<\infty$, arising from the definitions, are as follows.

$$
\begin{aligned}
E(a, \sigma(r), p(r)) & \subseteq V(a, \sigma(r), p(r)), \\
\mathcal{E}(a, \sigma(r)) & \subseteq \mathfrak{B}(a, \sigma(r)) .
\end{aligned}
$$

If $0 \leqq p(r) \leqq p_{1}(r)<\infty$, then

$$
\begin{aligned}
& V(a, \sigma(r), p(r)) \subseteq V\left(a, \sigma(r), p_{1}(\boldsymbol{r})\right) \subseteq \mathfrak{B}(a, \sigma(r)), \\
& E(a, \sigma(r), p(r)) \subseteq E\left(a, \sigma(r), p_{1}(\boldsymbol{r})\right) \subseteq \&(a, \sigma(r)) .
\end{aligned}
$$

If $0<\sigma_{1}(r)<\sigma(r)<\infty$ and $0 \leqq p(r) \leqq p_{1}(r)<\infty$, then

$$
\begin{aligned}
& V(a, \sigma(r), p(r)) \subseteq V\left(a, \sigma_{1}(r), p_{1}(r)\right), \\
& E(a, \sigma(r), p(r)) \subseteq E\left(a, \sigma_{1}(r), p_{1}(r)\right) .
\end{aligned}
$$

We may note explicitly that if $\sigma(r)$ is a nondecreasing function of $r$ in $0 \leqq r<R$, then, for $r_{0}<r_{1}<R$,

$$
\begin{aligned}
& r_{0} \in c \mathfrak{B}(a, \sigma(r)) \text { implies } \quad r_{1} \in c \mathfrak{B}(a, \sigma(r)), \quad . \\
& r_{0} \in c\left(\xi(a, \sigma(r)) \text { implies } r_{1} \in c \&(a, \sigma(r))\right. \text {. }
\end{aligned}
$$

Therefore, for a nondecreasing $\sigma(r)$ the sets $\mathfrak{B}(a, \sigma(r))$ and $\mathfrak{E}(a, \sigma(r))$ have the properties (8.2) and (8.21). If $\sigma(r)$ is not subject to this condition, and in particular if $\sigma(r)$ is a nonincreasing function of $r$ and is not constant, $R$ may be a limit point of all the sets $\mathfrak{B}(a, \sigma(r)), C \mathfrak{B}(a, \sigma(r)), \mathbb{E}(a, \sigma(r))$ and $C \mathbb{E}(a, \sigma(r))$.

The foregoing definitions are quite general in that the only restrictions on $\sigma(r)$ and $p(r)$ are that in the interval $0 \leqq r<R \leqq \infty$ they are both singlevalued, have only isolated ordinary discontinuities, and satisfy

$$
0<\sigma(r)<\infty ; \quad 0 \leqq p(r)<\infty .
$$

The sets defined furnish a useful descriptive apparatus. Wherever it is required to indicate the relative function, $1 /(f-a)$ may be written in place of $a$ (or $f$ in place of $a=\infty$ ).

9. The parabolic case. Iversen's Lemma. In the parabolic case $R=\infty$ we can establish relations complementary to (8.1) and (8.6). These follow immediately from the following theorem of Iversen which we designate as Iversen's Lemma.

IVERSEN'S Lemma( $\left.{ }^{15}\right)$. If $f(z)$ is meromorphic in $|z|<\infty$ and of finite

(15) Iversen $[5$, p. 5]. 
valency in an unbounded domain $G(a, \sigma)$, then, for any $\sigma_{1}<\sigma$, the domains $G\left(a, \sigma_{1}\right)$ contained in $G(a, \sigma)$ are finite in number and are all bounded.

Iversen's proof of this proposition depends upon the star theorem of Gross for parabolic surfaces $\left({ }^{16}\right)$. The corresponding problem for the hyperbolic case is certainly more difficult and we shall not enter upon it here.

It follows at once from Iversen's Lemma that in the parabolic case if $\sigma_{1}<\sigma$ and $0 \leqq p<\infty$,

$$
r \in V(a, \sigma, p) \text { implies } r \in E\left(a, \sigma_{1}, p\right) \text {. }
$$

For every $G(r, a, \sigma)$, whether bounded or unbounded, contains only bounded domains $G\left(a, \sigma_{1}\right)$ and these include all the $G\left(r, a, \sigma_{1}\right)$. Thus

$$
V(a, \sigma, p) \subseteq E\left(a, \sigma_{1}, p\right) .
$$

Similarly if $\sigma_{1}(r)<\sigma(r)$ and $0 \leqq p(r)<\infty$,

$$
V(a, \sigma(r), p(r)) \subseteq E\left(a, \sigma_{1}(r), p(r)\right) .
$$

\section{Deductions from Selberg's Theorem}

10. Selberg's Theorem. Selberg's Theorem was quoted in $\$ 1$. His argument in fact proves a significant theorem under a less drastic hypothesis. The contributions to $m(r, a)$ of the arcs of $|z|=r$ intercepted by the $G_{\nu}(r, a, \sigma)$ and the arcs outside these domains are estimated; all the other domains $G(a, \sigma)$ are ignored. It follows that Selberg's inequality (1.2) is satisfied for all $r \in E(a, \sigma, p)$. The hypothesis that every $G(a, \sigma)$ is bounded and that the valency of $f(z)$ does not exceed $p$ in any of these domains (or the equivalent hypothesis in terms of the $U(a, \sigma))$ is equivalent to the hypothesis that $E(a, \sigma, p)$ is the whole interval $0 \leqq r<R \leqq \infty$; and under this condition it follows that (1.2) is satisfied for all $r<R$. But to obtain a significant result it is a sufficient hypothesis that $R$ is a limit point of the set $E(a, \sigma, p)$. Selberg's theorem may thus be restated in the following more general form.

THEOREM A. If $f(z)$ is meromorphic in $|z|<R \leqq \infty$ and if for a given a there are positive numbers $\sigma$ and $p<\infty$ such that $R$ is a limit point of $E(a, \sigma, p)$, then

$$
m(r, a)<p(\log r+O(1))+\log ^{+} \frac{1}{\sigma}+O(1)
$$

for all $r \in E(a, \sigma, p)$.

It is not necessary to enter further into the proof of this theorem here since it will be seen to be a special case of Theorem II.

The same observations apply in the case of Teichmüller's inequality (1.1)

(16) Gross [1] and [2, V]. Also E.A.F. p. 276. 
which holds for all $r \in E(a, \sigma, 1)$ where $R \leqq \infty$ is a limit point of this set.

Subject to the general condition imposed that $T(r)$ is unbounded, two pairs of complementary corollaries follow from Theorem A.

Suppose first that $R$ is not a limit point of $C E(a, \sigma, p)$. Then $E(a, \sigma, p)$ contains an interval $r_{0}<r<R$ in which (10.1) is satisfied. It follows, on dividing (10.1) by $T(r)$, that $\Delta(a)=0$. We thus have the following corollary. then

Corollary A.1. If, for $\sigma>0$ and $p<\infty, R$ is not a limit point of $C E(a, \sigma, p)$,

$$
\Delta(a)=0 .
$$

The condition of this corollary is effectively the condition of Selberg's Theorem as stated in $\$ 1$, for no unbounded $G(a, \sigma)$ exists and in all but a finite number of these domains $f(z)$ is of valency not exceeding $p$.

From A.1 there follows the complementary corollary.

COROLlaRy A.2. If $\Delta(a)>0$ then, for any pair of numbers $\sigma>0$ and $p<\infty$, $R$ is a limit point of the set $C E(a, \sigma, p)$.

Secondly, from the less stringent condition of Theorem $\mathrm{A}$ that $R$ is a limit of $E(a, \sigma, p)$ it follows from (10.1) that $\delta(a)=0$. We thus have: then

Corollary A.3. If, for $\sigma>0$ and $p<\infty, R$ is a limit point of $E(a, \sigma, p)$,

$$
\delta(a)=0 .
$$

From A.3 there follows the complementary corollary:

CoRollaRY A.4. If $\delta(a)>0$ then, for any pair of numbers $\sigma>0$ and $p<\infty, R$ is not a limit point of the set $E(a, \sigma, p)$.

11. A qualitative theorem on exceptional values. General case. A general qualitative property of $f(z)-a$, for an exceptional value $a$, follows easily from Corollaries A.2 and A.4. This is expressed in the following theorem.

ThEOREM B. Suppose that $f(z)$ is meromorphic in $|z|<R \leqq \infty$ and $T(r)$ is unbounded. Then

(i) If $\Delta(a)>0$ we can find a nonincreasing function $\sigma(r) \rightarrow 0$ and a nondecreasing function $p(r) \rightarrow \infty$ as $r \rightarrow R$ such that $R$ is a limit point of the set $C E(a, \sigma(r), p(r))$.

(ii) If $\delta(a)>0$ we can find $\sigma(r)$ and $p(r)$ with the properties specified in (i) such that $R$ is not a limit point of $E(a, \sigma(r), p(r))$.

To prove (i) we use A.2. If $\Delta(a)>0$ and we choose two monotonic sequences

$$
\sigma_{1}>\sigma_{2}>\cdots>\sigma_{n}>\cdots, \lim _{n=\infty} \sigma_{n}=0
$$


and

$$
p_{1}<p_{2}<\cdots<p_{n}<\cdots, \lim _{n=\infty} p=\infty,
$$

it follows from A.2 that we can find an associated sequence

$$
r_{1}<r_{2}<\cdots<r_{n}<\cdots, \lim _{n=\infty} r_{n}=R,
$$

such that $r_{n} \in C E\left(a, \sigma_{n}, p_{n}\right)$. For in the case $R=\infty$ we may choose $r_{n}>2 r_{n-1}$ and in the case $R<\infty$ we may choose $\left(r_{n-1}+R\right) / 2<r_{n}<R$. If we now define $\sigma(r)$ by the equations

$$
\sigma(r)=\sigma_{n} \quad \text { for } r_{n} \leqq r<r_{n+1}
$$

and $p(r)$ by

$$
p(r)=p_{n} \quad \text { for } r_{n} \leqq r<r_{n+1}
$$

we see that the sequence $r_{n}$ belongs to $C E(a, \sigma(r), p(r))$, which thus has $R$ as a limit point. To prove (ii) we use A.4 in a similar way. We again choose sequences $\left\{\sigma_{n}\right\}$ and $\left\{p_{n}\right\}$ satisfying (11.1) and (11.2). It then follows from A.4 that if $\delta(a)>0$ there is an associated sequence $\left\{r_{n}\right\}$ satisfying (11.3) and such that $r$ in the interval $r_{n} \leqq r<R$ is in $C E\left(a, \sigma_{n}, p_{n}\right)$. If $\sigma(r)$ and $p(r)$ are again defined by (11.4) and (11.5) it follows that all $r$ in the interval $r_{1} \leqq r<R$ are in $C E(a, \sigma(r), p(r))$ so that $R$ is not a limit point of $E(a, \sigma(r), p(r))$ and the theorem is proved.

Now $C \&(a, \sigma(r)) \subseteq C E(a, \sigma(r), p(r))$ and for $r \in \mathbb{E}(a, \sigma(r)) \times C E(a, \sigma(r), p(r))$ we have $P(r, a, \sigma(r))>p(r)$ and since $p(r) \rightarrow \infty$ as $r \rightarrow R$ we have, as an immediate consequence of Theorem $B$, the following theorem on the valency $P(r, a, \sigma(r))$.

THEOREM B'. Suppose that $f(z)$ is meromorphic in $|z|<R \leqq \infty$ and $T(r)$ is unbounded. Then

(i) If $\Delta(a)>0$ we can find a nonincreasing function $\sigma(r) \rightarrow 0$ as $r \rightarrow R$ such that either (a) $R$ is a not limit point of $C \&(a, \sigma(r))$ and

$$
\limsup _{r=R} P(r, a, \sigma(r))=\infty ;
$$

or (b) $R$ is a limit point of $C \&(a, \sigma(r))$.

(ii) If $\delta(a)>0$ we can find a nonincreasing $\sigma(r) \rightarrow 0$ as $r \rightarrow R$ such that either (a) $R$ is not a limit point of $C \&(a, \sigma(r))$ and

$$
\lim _{r=R} P(r, a, \sigma(r))=\infty ;
$$

or (b) $R$ is a limit point of both $C \&(a, \sigma(r))$ and $\mathbb{E}(a, \sigma(r))$ and

$$
\lim _{r^{\prime}=R} P\left(r^{\prime}, a, \sigma\left(r^{\prime}\right)\right)=\infty,
$$


where $r^{\prime} \in \mathbb{E}(a, \sigma(r)$; or (c) $R$ is not a limit point of $\&(a, \sigma(r))$.

12. Qualitative theorem on exceptional values. Parabolic case $R=\infty$. It follows immediately from Iversen's Lemma that in the parabolic case $R=\infty$ the sets $E(a, \sigma, p)$ and $C E(a, \sigma, p)$ may be replaced by $V(a, \sigma, p)$ and $C V(a, \sigma, p)$ respectively in Theorem A and its corollaries. For, by (9.1) if $R$ is a limit point of $V(a, \sigma, p)$, then $R$ is also a limit point of $E(a, \sigma / 2, p)$ and (10.1) follows for all $r \in E(a, \sigma / 2, p)$ and, a fortiori, for all $r \in V(a, \sigma, p)$. Hence, in the parabolic case, in place of Theorem $B$ we have the following stronger theorem.

THEOREM C. Suppose that $f(z)$ is meromorphic in $|z|<\infty$. Then

(i) If $\Delta(a)>0$ we can find a nonincreasing $\sigma(r) \rightarrow 0$ and a nondecreasing $p(r) \rightarrow \infty$ as $r \rightarrow \infty$ such that $\infty$ is a limit point of $C V(a, \sigma(r), p(r))$. Hence the valency $P(r, a, \sigma(r))$ of the level $\sigma(r)$ relative to $f(z)$ and a satisfies

$$
\limsup _{\boldsymbol{r}=\infty} P(\boldsymbol{r}, a, \sigma(\boldsymbol{r}))=\infty \text {. }
$$

(ii) If $\delta(a)>0$ we can find $\sigma(r)$ and $p(r)$ with the properties specified in (i) such that $\infty$ is not a limit point of $V(a, \sigma(r), p(r)$.) Hence

$$
\lim _{r=\infty} P(r, a, \sigma(r))=\infty \text {. }
$$

13. Properties of asymptotic values of $f(z)$. It is interesting to compare the properties of deficient values established in Theorem B(ii) and Theorem C(ii) with properties of asymptotic values.

Consider first the general case where $f(z)$ is meromorphic in $|z|<R \leqq \infty$ and let $a$ be an asymptotic value of $f(z)$. There is then a curve $\Gamma(a)$ on which $|f(z)-a| \rightarrow 0$ as $|z| \rightarrow R . \Gamma(a)$ may be taken to be a Jordan curve having a finite number of intersections with a circumference $|z|=r<R$. Denote by $\Gamma_{r}(a)$ the unbounded arc of $\Gamma(a)$ lying outside $|z|=r$ and having only one end point on this circumference. By definition there is a nonincreasing function $\sigma(r) \rightarrow 0$ as $r \rightarrow R$ such that, for all $r$ in an interval $r_{0}<r<R,|f(z)-a|$ $<\sigma(r)$ on $\Gamma_{r}(a)$. Therefore $\Gamma_{r}(a)$ is contained in an unbounded domain $G(r, a, \sigma(r))$ and so $r \in C \&(a, \sigma(r))$. This proves the following theorem.

THEOREM D. If a is an asymptotic value of $f(z)$ meromorphic in $|z|<R \leqq \infty$, there is a nonincreasing $\sigma(r) \rightarrow 0$ as $r \rightarrow R$ such that $R$ is not a limit point of $\mathfrak{E}(a, \sigma(r))$.

For the parabolic case we prove a stronger theorem corresponding to Theorem C(ii). In this case $R=\infty$ and $\Gamma(a)$ tends to infinity. $\Gamma_{r}(a)$ and $\sigma(r)$ are defined as above. Choose a value $r_{1}$ so large that $\Gamma_{r_{1}}(a)$ is contained in an unbounded domain $G_{1}\left(r_{1}, a, \sigma\left(r_{1}\right)\right)=G_{1}$. The valency of $f(z)$ in $G_{1}$ is infinite. For if not, it follows from Iversen's Lemma that the domains $G\left(a, \sigma\left(r_{1}\right) / 2\right)$ contained in $G_{1}$ are bounded and finite in number. They are therefore all contained in a circle of radius $r_{2}>r_{1}$. Hence in the part of $G_{1}$ lying outside 
$|z|=r_{2}$ and therefore on $\Gamma_{r_{2}}(a)$ we have $|f(z)-a|>\sigma\left(r_{2}\right) / 2$, contrary to hypothesis. We have thus proved the following theorem.

TheOREM E. If $a$ is an asymptotic value of $f(z)$ meromorphic in $|z|<\infty$ there is a nonincreasing $\sigma(r) \rightarrow 0$ as $r \rightarrow \infty$ such that for all sufficiently large values of $r$

$$
P(r, a, \sigma(r))=\infty .
$$

The conclusions of Theorems $\mathrm{D}$ and $\mathrm{E}$ for an asymptotic value of $f(z)$ are stronger than those of Theorems $\mathrm{B}$ (ii) and $\mathrm{C}$ (ii) for a deficient value. It had been conjectured that in the parabolic case of a deficient value of a meromorphic function would also necessarily be an asymptotic value (F.M. p. 94; E.A.F. p. 258; Ullrich [1]). This conjecture was disproved first by Teichmüller [2] and soon afterward independently by Mme. Laurent-Schwartz [2]. The examples constructed by these authors, which are similar in character, show that a meromorphic function having an infinity of poles may have $\delta(a)>0$, where $a$ is finite or infinite and is not an asymptotic value. These examples do not, however, completely dispose of the problem even in the parabolic case. For no example is known of an integral function having $\delta(a)$ $>0$ for a finite $a$ which is not an asymptotic value. On this problem the reader is referred to a recent paper of Pflüger [1].

\section{INEQUALITIES DERIVED FROM THE MAIN THEOREM}

14. An inequality for a null level $\sigma(r)$ relative to $f(z)$ and $a$. The theorem and corollaries proved in this section, although trivial in themselves, are included to complete the formal pattern of the theory.

By definition $\sigma(r)$ is a null level relative to $f(z)$ and $a$ for $r=r_{0}$ if

$$
\left|f\left(r_{0} e^{i \phi}\right)-a\right| \geqq \sigma\left(r_{0}\right) ;
$$

We then write

$$
r_{0} \in V(a, \sigma(r), 0) \text { and } P\left(r_{0}, a, \sigma\left(r_{0}\right)\right)=0 .
$$

From (14.1)

$$
m\left(r_{0}, a\right) \leqq \log ^{+} \frac{1}{\sigma\left(r_{0}\right)}
$$

and we have the following theorem.

THEOREM I. If $f(z)$ is meromorphic in $|z|<R \leqq \infty$ and for a given $\sigma(r)$ the set $V(a, \sigma(r), 0)$ is not void, then

$$
m(r, a) \leqq \log ^{+} \frac{1}{\sigma(r)}
$$


for all $r \in V(a, \sigma(r), 0)$.

The inequality (14.2) is only significant if $\sigma(r)$ is small. For the purpose of our definitions $\sigma(r)$ has been subject only to the conditions of being singlevalued, finite, and positive in the interval $0 \leqq r<R$ in which it has only isolated ordinary discontinuities. From this point onwards we impose the additional condition

$$
\sigma\left(r^{\prime}\right) \leqq \sigma(r) \quad \text { for } 0 \leqq r<r^{\prime}<R \leqq \infty .
$$

Without restricting generality we may assume that either $\sigma(r)$ is equal to a constant $\sigma$ for all $r<R$ or that $\sigma(r) \rightarrow 0$ as $r \rightarrow R$. As a measure of the order of magnitude of $\sigma(r)$ in comparison with $T(r)$ we define the indices of $\sigma(r)$ with respect to $f(z)$ as follows.

The upper index $I^{*}(\sigma(r), f)$ and the lower index $I_{*}(\sigma(r), f)$ of $\sigma(r)$ with respect to $f(z)$ are defined respectively by $\left({ }^{17}\right)$

$$
I^{*}(\sigma(r), f)=\limsup _{r \rightarrow R} \frac{\log ^{+} 1 / \sigma(r)}{T(r)}
$$

and

$$
I_{*}(\sigma(r), f)=\liminf _{r=R} \frac{\log ^{+} 1 / \sigma(r)}{T(r)} .
$$

If $\lim _{r=R} \log ^{+}(1 / \sigma(r)) / T(r)$ exists it is the index of $\sigma(r)$ and is denoted by $I(\sigma(r), f)$. When there is no ambiguity these indices are written $I^{*}, I_{*}$, and $I$.

The alternative properties of the set $V(a, \sigma(r), 0)$ give four corollaries to Theorem I making the same pattern as the corollaries of Theorem A.

First suppose that $R$ is not a limit point of $C V(a, \sigma(r), 0)$. Then plainly $a$ is a Picard exceptional value of $f(z)$. For if $f(z)$ has an infinity of $a$-points $z_{n}(a)$, then $\left|z_{n}(a)\right| \in C V(a, \sigma(r), 0)$ and $\lim _{n=\infty}\left|z_{n}(a)\right|=R$. Hence we have $N(r, s)=O(\log r)$ and so, by (14.2),

$$
T(r)=m(r, a)+N(r, a)+O(1)<\log ^{+} \frac{1}{\sigma(r)}+O(\log r)+O(1)
$$

for all $r$ in an interval $r_{0}<r<R \leqq \infty$. Dividing (14.3) by $T(r)$ and proceeding to the limit we have the following corollary.

CoROLlaRy I.1. If, for a given $\sigma(r)$ tending to zero as $r \rightarrow R, R$ is not a limit point of $C V(a, \sigma(r), 0)$, then $a$ is a Picard value of $f(z)$ and

$$
\delta(a)=1 \leqq I_{*}(\sigma(r), f) .
$$

The complementary result is :

${ }^{(17)}$ In Collingwood [3] and [4] the upper and lower indices were denoted by $\bar{I}$ and $\underline{I}$ respectively. The notation $I^{*}$ and $I_{*}$ has been adopted here for typographical convenience. 
Corollary I.2. If $\sigma(r)$ is of lower index

$$
I_{*}(\sigma(r), f)<1,
$$

then $R$ is a limit point of $C V(a, \sigma(r), 0)$, that is, there is a sequence $r_{n} \rightarrow R$ such that the inequality $|f(z)-a|<\sigma(r)$ is satisfied at some points of $|z|=r_{n}$.

Now suppose that $R$ is a limit point of $V(a, \sigma(r), 0)$. The inequality (14.2) is satisfied for a sequence $r_{m} \rightarrow R$ and so

$$
\begin{aligned}
\delta(a) & =\liminf _{r=R} \frac{m(r, a)}{T(r)} \leqq \lim _{r_{m}=R} \frac{m\left(r_{m}, a\right)}{T\left(r_{m}\right)} \\
& \leqq \liminf _{r_{m}=R} \frac{\log ^{+} 1 / \sigma\left(r_{m}\right)}{T\left(r_{m}\right)} \leqq I^{*}(\sigma(r), f) .
\end{aligned}
$$

Hence we have:

CoRollary I.3. If $R$ is a limit point of $V(a, \sigma(r), 0)$, then

$$
\delta(a) \leqq I^{*}(\sigma(r), f) .
$$

We also have the complementary corollary:

CoRollaRy I.4. If $\delta(a)>0$ and $\sigma(r)$ is of upper index $I^{*}(\sigma(r), f)<\delta(a)$, then $R$ is not a limit point of $V(a, \sigma(r), 0)$, that is, for all $r$ in an interval $r_{0}<r<R \leqq \infty$ there are points of $|z|=r$ at which $|f(z)=a|<\sigma(r)$.

15. The main theorem. In IV we shall prove the following generalization of Selberg's Theorem. The theorem is in two parts (i) and (ii), the former giving the straightforward generalization of the inequality (10.1) valid without restriction; and the latter giving a sharper inequality subject to a topological condition on the domains $G(r, a, \sigma(r))$, designated condition $\left({ }^{*}\right)$, which is satisfied in important cases, including that of an unbounded holomorphic function.

THEOREM II. (i) Suppose that $f(z)$ is meromorphic in $|z|<R \leqq \infty$ and that for a given a there is a positive $\sigma(r)$ which is either constant or tends monotonically to zero as $r \rightarrow R$ and $a p(r)$ satisfying $0 \leqq p(r)<\infty$ for all $r<R$ such that $R$ is a limit point of the set $E=E(a, \sigma(r), p(r))$. Under these conditions we have, for all $r \in E$,

$$
m(r, a)<(\pi+\log r) p(r)+\log ^{+} \frac{1}{\sigma(r)}+O(1)
$$

in the parabolic case $R=\infty$; and

$$
m(r, a)<(\pi+o(1)) p(r)+\log ^{+} \frac{1}{\sigma(r)}+O(1)
$$


in the hyperbolic case $R<\infty$.

(ii) Suppose that in addition to the conditions specified in (i) the following condition is also satisfied, namely: $\left(^{*}\right)$ there are non-negative numbers $A<R$ and $B<R$ such that for any $r$ in $A<r<R$ none of the domains $G_{v}(r, a, \sigma(r))$, where $\nu \leqq \lambda(r)$, whether "bounded" or not, contains a closed curve which encloses the circumference $|z|=B$. Then, for $R \leqq \infty$ and all $r \in E$,

$$
m(r, a)<\frac{\pi}{2} p(r)+\log ^{+} \frac{1}{\sigma(r)}+O(1) .
$$

Clearly, condition $\left({ }^{*}\right)$ implies the weaker condition: $\left({ }^{* *}\right)$ there are nonnegative numbers $A<R$ and $B<R$ such that if $A<r \in E$ none of the domains $G_{\nu}(r, a, \sigma(r))$, where $\nu \leqq \lambda(r)$, contains a closed curve which encloses the circumference $|z|=B$.

We shall in fact prove that, for $R \leqq \infty,(15.3)$ is satisfied for all $r \in E$ if condition $\left(^{* *}\right)$ is satisfied; and this result includes (ii). But for the applications we have in view here, the stronger condition $\left({ }^{*}\right)$ is the one generally required.

Theorem A follows from II (i) on putting $\sigma(r)=\sigma$ and $p(r)=p$; and the other known results emerge as special cases of Theorem II, or of its more general form with the condition $\left({ }^{* *}\right)$ in place of $(*)$, on assigning suitable properties to $\sigma(r), p(r)$, and the set $E$.

Cases in which condition $\left(^{*}\right)$ is satisfied include the following:

$(\alpha)$ If $f(z)$ is holomorphic in $|z|<R, T(r)$ is unbounded and $a \neq \infty$. For suppose the contrary. Then, given any positive $A<R$ and $B<R$, there is an $r$ in $A<r<R$ such that $|z|=B$ is enclosed in a closed curve contained in a domain $G(r, a, \sigma(r))$. But this domain if "bounded" is simply-connected and if "unbounded" has no closed contour. It therefore contains the domain $|z| \leqq B$. Hence $|f(z)-a|<\sigma(r)$ in $|z| \leqq B$. If $\sigma(r) \rightarrow 0$ as $r \rightarrow R$ it follows that $f(z) \equiv a$ in $|z|<R$. And if $\sigma(r)=\sigma$ we have $|f(z)-a|<\sigma$ in $|z| \leqq B$; and so, since $B<R$ but is otherwise arbitrary, $|f(z)|<|a|+\sigma$ for $|z|<R$ and consequently $T(r)=O(1)$, which is contrary to hypothesis.

( $\beta)$ If $f(z)$ has an asymptotic value $b \neq a$ and there is an $r_{1}<R$ such that $\sigma(r)<|b-a|$ for all $r$ in $r_{1}<r<R$. For there is a curve $\Gamma(b)$ on which $|z| \rightarrow R$ and $|f(z)-b|<|b-a|-\sigma\left(r_{1}\right)$ and thus also $|f(z)-a|>\sigma\left(r_{1}\right)$. Let $|z|=r_{2}$ intersect $\Gamma(b)$ and put $A=r_{1}$ and $B=r_{2}$. Then any closed curve enclosing $|z|=B$ intersects $\Gamma(b)$ and so cannot belong to any $G(r, a, \sigma(r))$ where $A<r<R$.

( $\gamma$ ) More generally, if there is a curve in $|z|<R$ on which $|z| \rightarrow R$ and $|f(z)-a|>\sigma\left(r_{1}\right)$ for some $r_{1}<R$.

The weaker condition (**) is satisfied if, for $r \in E$, the "bounded" domains $G(r, a, \sigma(r))$ are all simply-connected. For, as in $(\alpha)$ above, if $|z|=B$ is enclosed in a closed curve belonging to a domain $G(r, a, \sigma(r))$, where $r \in E$, it follows that $|f(z)-a|<\sigma(r)$ for all $z$ in $|z| \leqq B$, so that, since $B<R$ but is otherwise arbitrary, $T(r)=O(1)$.

As an immediate application of this last remark we observe that in every 
case all the $G(r, a, \sigma(r))$ are simply-connected for $r<E(a, \sigma(r), 1)$ so that if $p(r)=1,(15.3)$ is always satisfied; and this gives the inequality

$$
m(r, a)<\log ^{+} \frac{1}{\sigma(r)}+O(1)
$$

for $r<E(a, \sigma(r), 1)$. This result generalizes a previous schlicht condition theorem given by the writer for the parabolic case $R=\infty\left({ }^{18}\right)$. For a constant $\sigma(r)=\sigma,(15.1)$ reduces to Teichmüller's inequality.

The inequality (15.3) also holds if, for $r \in E(a, \sigma(r), p(r))$, the sole branch point of each $U_{\nu}(r, a, \sigma(r))$ is an algebraic point of order not exceeding $p(r)-1$ over $w=a . U_{\nu}(r, s, \sigma(r))$ and $G_{\nu}(r, a, \sigma(r))$ are then simply-connected and the only zero of $f^{\prime}(z)$ in $G_{\nu}(r, a, \sigma(r))$ is at the multiple $a$-point if $p_{\nu}>1$. If this condition is satisfied for a constant $\sigma(r)=\sigma$, then

$$
m(r, a)<\frac{\pi}{2} p(r)+O(1)
$$

for $r \in E(a, \sigma(r), p(r)):$ and if $R$ is not a limit point of $C E(a, \sigma(r), p(r))$ and $p(r)=o(T(r))$ we have $\delta(a)=\Delta(a)=0$. This generalizes and sharpens a previous result of Yosida [1] and Kakutani[1].

16. Corollaries to Theorem II. The parabolic case $R=\infty$. The corollaries conform to the pattern already established. It is convenient to separate the parabolic and hyperbolic cases and we begin with the former. In this case $f(z)$ is meromorphic in $|z|<\infty$ and we have seen ((9.2) above) that, for a given $\sigma(r)$ and $p(r), V(a, \sigma(r), p(r)) \subseteq E(a, \sigma(r) / 2, p(r))$. Thus if $V(a, \sigma(r), p(r))$ has $R$ as a limit point, so has $E(a, \sigma(r) / 2, p(r))$. Also, if for $r>A$ no domain $G_{\nu}(r, a, \sigma(r))$, whether or not it is "bounded," contains a closed curve enclosing the circumference $|z|=B$, the same condition is satisfied by the domains $G_{\mu}(r, a, \sigma(r) / 2)$ contained in them, all of which are "bounded" for $r \in V(a, \sigma(r), p(r))$. We may therefore replace the set $E(a, \sigma(r), p(r))$ by $V(a, \sigma(r), p(r))$ in the enunciation of both Theorem II and of condition (**) if $R=\infty$. This rather more general form of the theorem gives the four corollaries which follow. The hypothesis common to all of them is that $f(z)$ is meromorphic in $|z|<\infty$ while $\sigma(r)$ is either constant or tends monotonically to zero as $r \rightarrow \infty, 0 \leqq p(r)<\infty$, and $a$ is any complex number not excluding infinity.

Suppose first that $\infty$ is not a limit point of $C V(a, \sigma(r), p(r))$, that is, $P(r, a, \sigma(r)) \leqq p(r)$ for all sufficiently large $r$. The inequalities (15.1) and (15.3) thus hold for all sufficiently large $r$ and, on dividing these inequalities by $T(r)$, we obtain the inequalities for $\Delta(a)$ and $\delta(a)$ given in the following corollary.

(18) Collingwood [2]. The inequality (2) of this note is equivalent to $m(r, a)<\log ^{+}(1 / \sigma(r))$ $+m\left(r, f^{\prime} /(f-a)\right)+O(\log r)$ since $\log \left(1 / \sigma_{f}(r)\right) \leqq \log ^{+}(1 / \sigma(r)), \sigma_{f}(r)$ being the function there defined. 
CoRollaRy II. 1.1. If, for a given $\sigma(r)$ and $p(r), \infty$ is not a limit point of $C V(a, \sigma(r), p(r))$ (that is, if $P(r, a, \sigma(r)) \leqq p(r)$ for all sufficiently large $r)$, then in general,
(a) $\Delta(a) \leqq \limsup _{r=\infty} \frac{p(r) \log r}{T(r)}+I^{*}(\sigma(r), f)$,
(b) $\delta(a) \leqq \limsup _{r=\infty} \frac{p(r) \log r}{T(r)}+I_{*}(\sigma(r), f)$,
(c) $\delta(a) \leqq \liminf _{r=\infty} \frac{p(r) \log r}{T(r)}+I^{*}(\sigma(r), f) ;$

or, under condition $\left(^{*}\right)$,
(a) $\Delta(a) \leqq \frac{\pi}{2} \limsup _{r=\infty} \frac{p(r)}{T(r)}+I^{*}(\sigma(r), f)$,
(b) $\delta(a) \leqq \frac{\pi}{2} \limsup _{r=\infty} \frac{p(r)}{T(r)}+I_{*}(\sigma(r), f)$,
(c) $\delta(a) \leqq \frac{\pi}{2} \liminf _{r=\infty} \frac{p(r)}{T(r)}+I^{*}(\sigma(r), f)$.

The complementary corollary to this is:

COROLLARY II. 1.2. If, for a given $\sigma(r)$ and $p(r)$, in the general case any of the inequalities
(a) $\Delta(a)>\limsup \frac{p(r) \log r}{T(r)}+I^{*}(\sigma(r), f)$,
(b) $\delta(a)>\limsup \frac{p(r) \log r}{T(r)}+I_{*}(\sigma(r), f)$,
(c) $\delta(a)>\liminf _{r=\infty} \frac{p(r) \log r}{T(r)}+I^{*}(\sigma(r), f)$

is satisfied; or under condition $\left(^{*}\right)$ if any of the inequalities
(a) $\Delta(a)>\frac{\pi}{2} \limsup _{r=\infty} \frac{p(r)}{T(r)}+I^{*}(\sigma(r), f)$,
(b) $\delta(a)>\frac{\pi}{2} \limsup _{r=\infty} \frac{p(r)}{T(r)}+I_{*}(\sigma(r), f)$,
(c) $\delta(a)>\frac{\pi}{2} \liminf _{r=\infty} \frac{p(r)}{T(r)}+I^{*}(\sigma(r), f)$ 
is satisfied, then $\infty$ is a limit point of $C V(a, \sigma(r), p(r)$ ) (that is, $P(r, a, \sigma(r)$ ) $>p(r)$ for some large values of $r)$.

We obtain a second complementary pair of corollaries to Theorem II with the condition that $\infty$ is a limit point of $V(a, \sigma(r), p(r))$ but with no condition on $C V(a, \sigma(r), p(r))$. We then have from (15.1)

$$
\begin{aligned}
\delta(a) & =\liminf _{r=\infty} \frac{m(r, a)}{T(r)} \leqq \liminf _{r^{\prime}=\infty} \frac{m\left(r^{\prime}, a\right)}{T\left(r^{\prime}\right)} \\
& \leqq \liminf _{r^{\prime}=\infty} \frac{p\left(r^{\prime}\right) \log r^{\prime}}{T\left(r^{\prime}\right)}+I^{*}(\sigma(r), f) \\
& \leqq \limsup _{r=\infty} \frac{p(r) \log r}{T(r)}+I^{*}(\sigma(r), f)
\end{aligned}
$$

where $r^{\prime}$ denotes a value of $r$ in $V(a, \sigma(r), p(r))$. Similarly from (15.3)

$$
\delta(a) \leqq \frac{\pi}{2} \limsup _{r=\infty} \frac{p(r)}{T(r)}+I^{*}(\sigma(r), f) .
$$

Hence we have:

CoRollary II. 1.3. If, for a given $\sigma(r)$ and $p(r), \infty$ is a limit point of $V(a, \sigma(r), p(r))$ (that is, if $P(r, a, \sigma(r)) \leqq p(r)$ for some large values of $r)$, then, in general,

$$
\delta(a) \leqq \limsup _{r=\infty} \frac{p(r) \log r}{T(r)}+I^{*}(\sigma(r), f)
$$

or, under condition $(*)$,

$$
\delta(a) \leqq \frac{\pi}{2} \limsup _{r=\infty} \frac{p(r)}{T(r)}+I^{*}(\sigma(r), f) .
$$

The complementary corollary is :

CoROLlaRy II. 1.4. If, for any given $\sigma(r)$ and $p(r)$, the inequality

$$
\delta(a)>\limsup _{r=\infty} \frac{p(r) \log r}{T(r)}+I^{*}(\sigma(r), f)
$$

or, under condition $\left(^{*}\right)$, the inequality

$$
\delta(a)>\frac{\pi}{2} \limsup _{r=\infty} \frac{p(r)}{T(r)}+I^{*}(\sigma(r), f)
$$

is satisfied, then $\infty$ is not a limit point of $V(a, \sigma(r), p(r)$ ) (that is, $P(r, a, \sigma(r)$ ) $>p(r)$ for all large values of $r)$. 
17. A theorem concerning $P(r, a, \sigma(r))$ in the parabolic case. For a given nonincreasing $\sigma(r)$ and an arbitrary value $a$ the valency $P(r, a, \sigma(r))$ of $\sigma(r)$ regarded as a level relative to $f(z)$ and $a$ may be finite or infinite for finite values of $r$. We shall prove the following theorem.

THEOREM III. If $f(z)$ is meromorphic in $|z|<\infty, \sigma(r)$ is a nonincreasing function of $r$ and $a$ is any complex number, then the valency $P(r, a, \sigma(r))$ of $\sigma(r)$ regarded as a level relative to $f(z)$ and a satisfies the inequalities

$$
\begin{aligned}
& \text { (a) } \Delta(a) \leqq \limsup _{r=\infty} \frac{P(r, a, \sigma(r)) \log r}{T(r)}+I^{*}(\sigma(r), f), \\
& \text { (b) } \delta(a) \leqq \limsup _{r=\infty} \frac{P(r, a, \sigma(r)) \log r}{T(r)}+I_{*}(\sigma(r), f), \\
& \text { (c) } \delta(a) \leqq \liminf _{r=\infty} \frac{P(r, a, \sigma(r)) \log r}{T(r)}+I^{*}(\sigma(r), f)
\end{aligned}
$$

or, under the condition $\left({ }^{*}\right)$, satisfies the inequalities

$$
\begin{aligned}
& \text { (a) } \Delta(a) \leqq \frac{\pi}{2} \limsup _{r=\infty} \frac{P(r, a, \sigma(r))}{T(r)}+I^{*}(\sigma(r), f), \\
& \text { (b) } \delta(a) \leqq \frac{\pi}{2} \limsup _{r=\infty} \frac{P(r, a, \sigma(r))}{T(r)}+I_{*}(\sigma(r), f), \\
& \text { (c) } \delta(a) \leqq \frac{\pi}{2} \liminf _{r=\infty} \frac{P(r, a, \sigma(r))}{T(r)}+I^{*}(\sigma(r), f) .
\end{aligned}
$$

The proof follows from Corollaries II. 1.2. and II. 1.4. It is enough to prove the inequalities (17.1). Of these $(17.1)(\mathrm{a})$ and $(17.1)(\mathrm{b})$ are deduced from Corollary II. 1.2. (17.1) (a) is trivial if $\Delta(a)=0$ or if $I^{*}(\sigma(r), f) \geqq \Delta(a)>0$. We therefore assume $0 \leqq I^{*}(\sigma(r), f)<\Delta(a)$. Now in Corollary II. 1.2, $p(r)$ is arbitrary so we may put

$$
\lim _{r=\infty} \frac{p(r) \log r}{T(r)}=\Delta(a)-I^{*}(\sigma(r), f)-\epsilon,
$$

where $\epsilon>0$ is arbitrarily small. Then by $(16.3)(a), \infty$ is a limit point of $C V(a, \sigma(r), p(r))$ and so, if we write $r^{\prime} \in C V(a, \sigma(r), p(r))$, we have, for all sufficiently large $r^{\prime}, P\left(r^{\prime}, a, \sigma\left(r^{\prime}\right)\right)>p\left(r^{\prime}\right)$ and so

$$
\begin{aligned}
\lim \sup \frac{P(r, a, \sigma(r)) \log r}{r=\infty} & \geqq \limsup _{r^{\prime}=\infty} \frac{P\left(r^{\prime}, a, \sigma\left(r^{\prime}\right)\right) \log r^{\prime}}{T\left(r^{\prime}\right)} \\
& \geqq \limsup _{r^{\prime}=\infty} \frac{p\left(r^{\prime}\right) \log r^{\prime}}{T\left(r^{\prime}\right)}=\Delta(a)-I^{*}(\sigma(r), f)-\epsilon .
\end{aligned}
$$


But $\epsilon>0$ is arbitrary and so (17.1)(a) follows. To prove (17.1)(b), which is trivial if $\delta(a)=0$ or if $I^{*}(\sigma(r), f) \geqq \delta(a)>0$, we assume

$$
0 \leqq I_{*}(\sigma(r), f)<\delta(a)
$$

and put

$$
\lim _{r=\infty} \frac{p(r) \log r}{T(r)}=\delta(a)-I_{*}(\sigma(r), f)-\epsilon .
$$

Then, by (16.3)(b), $\infty$ is again a limit point of $C V(a, \sigma(r), p(r))$ and so, exactly as above,

$$
\limsup _{r=\infty} \frac{P(r, a, \sigma(r)) \log r}{T(r)} \geqq \lim _{r^{\prime}=\infty} \frac{p\left(r^{\prime}\right) \log r^{\prime}}{T\left(r^{\prime}\right)}=\delta(a)-I_{*}(\sigma(r), f)-\epsilon
$$

which gives (17.1)(b) since $\epsilon>0$ is arbitrary.

To prove $(17.1)$ (c) we use Corollary II. 1.4. The inequality is trivial if $\delta(a)=0$ or if $I^{*}(\sigma(r), f) \geqq \delta(a)>0$ and so we assume

$$
0 \leqq I^{*}(\sigma(r), f)<\delta(a)
$$

and put

$$
\lim _{r=\infty} \frac{p(r) \log r}{T(r)}=\delta(a)-I^{*}(\sigma(r), f)-\epsilon
$$

It then follows from (16.7) that $P(r, a, \sigma(r))>p(r)$ for all large $r$ and so

$$
\lim _{r=\infty} \frac{P(r, a, \sigma(r)) \log r}{T(r)} \geqq \lim _{r=\infty} \frac{p(r) \log r}{T(r)}=\delta(a)-I^{*}(\sigma(r), f)-\epsilon
$$

and, $\epsilon>0$ being arbitrary, (17.1)(c) is proved. The inequalities (17.2) are deduced in precisely the same way from (16.4) and (16.8). The theorem is therefore proved.

18. Deductions from Theorem III. Three kinds of conclusions may be drawn from the inequalities of Theorem III, the known results emerging as special cases.

First, they give upper bounds for the deficiencies $\Delta(a)$ and $\delta(a)$ in terms of the indices of $\sigma(r)$ and the corresponding valency $P(r, a, \sigma(r))$. In particular if, for a $\sigma(r)$ of index $I(\sigma(r), f)=0$, the valency $P(r, a, \sigma(r))=o((T(r) / \log r)$, or under condition $\left(^{*}\right)$ if $P(r, a, \sigma(r))=o(T(r))$, then $\Delta(a)=0$; and if $\lim \inf _{r=\infty} P(r, a \sigma(r)) \log r / T(r)=0$, or, under condition $\left(^{*}\right)$, if lim $\inf _{r=\infty}$ $P(r, a, \sigma(r)) / T(r)=0$, then $\delta(a)=0$. These conditions are satisfied for fixed $\sigma$ and $p$ so that Corollaries A1 and A3 in the parabolic case are special cases of our new result.

Secondly, Theorem III gives lower bounds for the indices $I^{*}(\sigma(r), f)$ and 
$I_{*}(\sigma(r), f)$ in terms of the deficiencies $\Delta(a)$ and $\delta(a)$ and valency $P(r, a, \sigma(r))$. The results, which are immediate, may be stated as the following corollary.

CoROLlary III.1. If $\Delta(a)>0$ and if

$$
\underset{r=\infty}{\limsup } \frac{P(r, a, \sigma(r)) \log r}{T(r)} \leqq \eta \Delta(a), \quad 0 \leqq \eta<1,
$$

or, under condition $(*)$,

$$
\frac{\pi}{2} \limsup _{r=\infty} \frac{P(r, a, \sigma(r))}{T(r)} \leqq \eta \Delta(a)
$$

then, by (17.1)(a) and (17.2)(a),

$$
I^{*}(\sigma(r), f) \geqq(1-\eta) \Delta(a)>0 .
$$

Similarly, if $\delta(a)>0$ and

$$
\limsup _{r=\infty} \frac{P(r, a, \sigma(r)) \log r}{T(r)} \leqq \eta \delta(a)
$$

or, under condition $(*)$,

$$
\frac{\pi}{2} \limsup _{r=\infty} \frac{P(r, a, \sigma(r))}{T(r)} \leqq \eta \delta(a)
$$

then, by (17.1)(b) and (17.2(b),

$$
I_{*}(\sigma(r), f) \geqq(1-\eta) \delta(a) .
$$

Thirdly, Theorem III gives minorizing conditions for $P(r, a, \sigma(r))$ in terms of the deficiencies $\Delta(a)$ and $\delta(a)$ and the indices $I^{*}(\sigma(r), f)$ and $I_{*}(\sigma(r), f)$. We have immediately:

CoRollary III.2 (i). If $\Delta(a)>0$ and we choose $\sigma(r) \rightarrow 0$ as $r \rightarrow \infty$ such that

$$
I^{*}(\sigma(r), f) \leqq \theta \Delta(a), \quad 0 \leqq \theta<1,
$$

then, by (17.1)(a),

$$
\limsup _{r=\infty} \frac{P(r, a, \sigma(r)) \log r}{T(r)} \geqq(1-\theta) \Delta(a)>0
$$

in general; or by (17.2)(a),

$$
\frac{\pi}{2} \limsup _{r=\infty} \frac{P(r, a, \sigma(r))}{T(r)} \geqq(1-\theta) \Delta(a)>0
$$

under condition $(*)$. 
(ii) Similarly, if $\delta(a)>0$ and we choose $\sigma(r)$ such that

$$
I^{*}(\sigma(r), f) \leqq \theta \delta(a),
$$

then, by $(17.1)(c)$,

$$
\liminf _{r=\infty} \frac{P(r, a, \sigma(r)) \log r}{T(r)} \geqq(1-\theta) \delta(a)>0
$$

in general; or, by (17.2)(c),

$$
\frac{\pi}{2} \liminf _{r=\infty} \frac{P(r, a, \sigma(r))}{T(r)} \geqq(1-\theta) \delta(a)>0
$$

under condition $\left(^{*}\right)$.

This corollary is much sharper than Theorem $\mathrm{C}$, which it includes as a special case. If we put $\theta=0$ so that $I(\sigma(r), f)=0$, we have in general

$$
\begin{gathered}
\underset{r=\infty}{\lim \sup } \frac{P(r, a, \sigma(r)) \log r}{T(r)} \geqq \Delta(a), \\
\liminf _{r=\infty} \frac{P(r, a, \sigma(r)) \log r}{T(r)} \geqq \delta(a)
\end{gathered}
$$

or, under condition $\left(^{*}\right)$,

$$
\begin{aligned}
& \frac{\pi}{2} \limsup _{r=\infty} \frac{P(r, a, \sigma(r))}{T(r)} \geqq \Delta(a), \\
& \frac{\pi}{2} \liminf _{r=\infty} \frac{P(r, a, \sigma(r))}{T(r)} \geqq \delta(a) .
\end{aligned}
$$

For a constant $\sigma(r)=\sigma$ the lower order of $P(r, a, \sigma(r))$ as given by the inequality (18.5) is best possible within a constant factor. This is shown by the function of Mme. Laurent-Schwartz referred to in $\$ 13$. The function in question, defined by

$$
f(z)=\prod_{n=1}^{\infty}\left(\frac{1+z / 4^{n}}{1-z / 4^{n}}\right)^{(-2)^{n}}
$$

is meromorphic in $|z|<\infty$, of order $1 / 2$, and has $\delta(0)=\delta(\infty)>0$, while neither 0 nor $\infty$ is an asymptotic value. Moreover, since $|f(z)|=1$ on the imaginary axis we have case $(\gamma)$ of $\$ 15$ and condition $\left(^{*}\right)$ is satisfied for $a=0$ or $\infty$ and $\sigma<1$. It is easily shown from the calculations of Mme. LaurentSchwartz $\left({ }^{19}\right)$ that for a fixed $\sigma<1$ and all values of $r$

(19) Laurent-Schwartz [2]. A sketch of the proof, using the notation of the note cited, is added for completeness.

There is a family of closed curves $\gamma_{n}$ tending uniformly to infinity on which $|f(z)|=1+o(1)$. 


$$
\frac{P(r, 0, \sigma)}{T(r)}=\frac{P(r, \infty, \sigma)}{T(r)}<k<\infty
$$

which proves our assertion. We conclude that under condition $\left(^{*}\right)$ Theorem III gives results which are substantially best possible.

19. Remark on a theorem of Macintyre. There is a suggestive analogy between Corollary III.2 (ii) and a theorem proved by Macintyre $\left({ }^{20}\right.$ ) in connection with J. M. Whittaker's concept of flat regions. The theorem in question is restricted to the parabolic case and finite order. It may be stated as follows.

Suppose that $f(z)$, meromorphic in $|z|<\infty$; is of finite order $\rho>0$ and let $\rho^{\prime} \geqq \rho$ be such that

$$
T(r)=o\left(\frac{r^{\rho^{\prime}}}{\log r}\right)
$$

Then if $\delta(a)>0$ and

$$
I^{*}(\sigma(r), f)<1 / e
$$

there is a sequence of points $\xi_{\mu}$, where $\lim _{\mu=\infty}\left|\xi_{\mu}\right|=\infty$, such that the circles $C_{\mu}$ defined by

$$
\left|z-\xi_{\mu}\right|<r_{\mu}, \quad w h e r e \quad \lim _{\mu=\infty} r_{\mu}\left|\xi_{\mu}\right|^{-1+\rho^{\prime} / 2}=\infty \text {, }
$$

are all contained in domains $G\left(\left|\xi_{\mu}\right|, a, \sigma\left(\left|\xi_{\mu}\right|\right)\right)$.

Here we have a minorizing condition not, like (18.4) and (18.41), on the maximum valency of $f(z)$ in the domains $G(r, a, \sigma(r))$ for all large $r$ but on the maximum dimensions of these domains for some large $r$.

20. The hyperbolic case $R<\infty$. We shall as usual take $R=1$. To obtain significant results from Theorem II we must confine ourselves to functions $f(z)$ meromorphic in $|z|<1$ for which $T(r)$ is unbounded. For any function

Hence, if we put $\sigma(r)=\sigma<1$, every $G(r, 0, \sigma)$ lies between successive curves $\gamma_{n}$ and $\gamma_{n+1}$ so that $P(r, 0, \sigma)$ is finite. Now $\gamma_{n+1}$ is the magnification of $\gamma_{n}$ by the factor 4 and it follows from symmetry considerations that the $G(r, 0, \sigma)$ and the $G(r, \infty, \sigma)$ are simply-connected. $P(r, 0, \sigma)$ is the number of zeros in $G(r, 0, \sigma)$. These zeros are concentrated at a point $\pm 4^{\Lambda_{n}}$ and the corresponding $P(r, 0, \sigma)=2^{\Lambda_{n}}$. Now $G(r, 0, \sigma)$ lies wholly within a circle $|z|<k r$, where $k$ is a constant, and so $P(r, 0, \sigma)<(k r)^{1 / 2}$; or, writing $r=4^{\nu} \rho$, where $1 \leqq \rho<4, P(r, 0, \sigma)<2^{\nu}(k \rho)^{1 / 2}$.

It is shown in the note cited that $N(r, 0)=N(r, \infty)=2^{\nu+1}(1-o(1) \log 4 \rho$ and hence $\left.P(r, 0, \sigma) / T(r)<P(r, 0, \sigma) / N(r, 0)<(k \rho)^{1 / 2}\right) / 2(1-o(1) \log 4 \rho<\infty$. The same argument applies to $P(r, \infty, \sigma)$; and so $(18.6)$ is proved.

It will be noted that $f(z)$ is a function for which $\mathfrak{E}(a, \sigma)$ and $\mathfrak{E}(a, 1 / \sigma)$ both have $\infty$ as a limit point. We may also note that an integral function of order less than $1 / 2$ has $\infty$ as a limit point of $\mathfrak{E}(a, \sigma)$ for all finite $a$ and $\sigma$.

${ }_{20}$ Macintyre [1, p. 273, Theorem 2]. 
of this class the set of exceptional values $a$ for which $\Delta(a)>0$ does not contain a continuum $\left({ }^{21}\right)$. Hence, since by hypothesis $\sigma(r)>0$ for all $r<1$, there is always at least one domain $U(a, \sigma(r))$ in the hyperbolic surface $S$.

Iversen's Lemma is no longer applicable so that the set $E(a, \sigma(r), p(r))$ cannot be replaced in Theorem II by $V(a, \sigma(r), p(r))$ as was done in the parabolic case. We have to work with the sets $E$ and $C E$. Subject to this, four corollaries to Theorem II for the hyperbolic case follow just as in $\$ 16$ for the parabolic case. The hypothesis common to all of them is that $f(z)$ is meromorphic in $|z|<1, T(r)$ is unbounded, $\sigma(r)$ is either a positive constant or a positive function tending monotonically to zero as $r \rightarrow 1,0 \leqq p(r)<\infty$, and a is any complex number not excluding infinity.

COROLLARY II. 2.1. If for a given $\sigma(r)$ and $p(r), r=1$ is not a limit point of $C E(a, \sigma(r), p(r))$ (that is, if $\sigma(r)$ is a closed level relative to $f(z)$ and a with $P(r, a, \sigma(r)) \leqq p(r)$ for all sufficiently large $r<1)$, then in general

$$
\begin{aligned}
& \text { (a) } \Delta(a) \leqq \pi \lim \sup _{r=1} \frac{p(r)}{T(r)}+I^{*}(\sigma(r), f), \\
& \text { (b) } \delta(a) \leqq \pi \lim \sup _{r=1} \frac{p(r)}{T(r)}+I_{*}(\sigma(r), f), \\
& \text { (c) } \delta(a) \leqq \pi \lim \inf _{r=1} \frac{p(r)}{T(r)}+I^{*}(\sigma(r), f)
\end{aligned}
$$

or, under condition $(*)$,
(a) $\Delta(a) \leqq \frac{\pi}{2} \limsup _{r=1} \frac{p(r)}{T(r)}+I^{*}(\sigma(r), f)$,
(b) $\delta(a) \leqq \frac{\pi}{2} \lim \sup _{r=1} \frac{p(r)}{T(r)}+I_{*}(\sigma(r), f)$,
(c) $\delta(a) \leqq \frac{\pi}{2} \liminf _{r=1} \frac{p(r)}{T(r)}+I^{*}(\sigma(r), f)$.

COROLLARY II. 2.2. If for a given $\sigma(r)$ and $p(r)$ any of the inequalities
(a) $\Delta(a)>\pi \lim \sup _{r=1} \frac{p(r)}{T(r)}+I^{*}(\sigma(r), f)$,
(b) $\delta(a)>\pi \lim \sup _{r=1} \frac{p(r)}{T(r)}+I_{*}(\sigma(r), f)$,
(c) $\delta(a)>\pi \liminf _{r=1} \frac{p(r)}{T(r)}+I^{*}(\sigma(r), f)$

${ }^{21}$ F.M. p. 155. 
in the general case or, under condition (*), any of the inequalities
(a) $\Delta(a)>\frac{\pi}{2} \limsup _{r=1} \frac{p(r)}{T(r)}+I^{*}(\sigma(r), f)$,
(b) $\delta(a)>\frac{\pi}{2} \lim \sup _{r=1} \frac{p(r)}{T(r)}+I_{*}(\sigma(r), f)$,
(c) $\delta(a)>\frac{\pi}{2} \liminf _{r=1} \frac{p(r)}{T(r)}+I^{*}(\sigma(r), f)$

is satisfied, then $r=1$ is a limit point of $C E(a, \sigma(r), p(r))$.

COROllaRY II. 2.3. If, for a given $\sigma(r)$ and $p(r), r=1$ is a limit point of $E(a, \sigma(r), p(r))$, then in general

$$
\delta(a) \leqq \pi \lim \sup \frac{p(r)}{T(r)}+I^{*}(\sigma(r), f)
$$

or, under condition $\left(^{*}\right)$,

$$
\delta(a) \leqq \frac{\pi}{2} \lim \sup _{r=1} \frac{p(r)}{T(r)}+I^{*}(\sigma(r), f) .
$$

COROLlARY II. 2.4. If, for any given $\sigma(r)$ and $p(r)$, the inequality

$$
\delta(a)>\pi \lim \sup \frac{p(r)}{T(r)}+I^{*}(\sigma(r), f)
$$

in the general case or, under condition $\left(^{*}\right)$, the inequality

$$
\delta(a)>\frac{\pi}{2} \lim \sup _{r=1} \frac{p(r)}{T(r)}+I^{*}(\sigma(r), f)
$$

is satisfied, then $r=1$ is not a limit point of $E(a, \sigma(r), p(r))$.

We may note in connection with Corollary II. 2.3 that Valiron [2] gives an example of a function regular in $|z|<1$ and of infinite order for which $\mathscr{E}(a, \sigma)$ has $r=1$ as a limit point for all finite $a$ and $\sigma$.

21. Analogue of Theorem III in the hyperbolic case. Since we have no theorem for the hyperbolic case corresponding to Iversen's Lemma we cannot extend Theorem III to this case. But we shall prove the following analogous theorem.

Theorem IV. Suppose that $f(z)$ is meromorphic in $|z|<1, T(r)$ is unbounded, $\sigma(r)$ is a nonincreasing function of $r$ in $0 \leqq r<1$, and $a$ is any complex number. Then either

(i) $r=1$ is not a limit point of $C \&(a, \sigma(r))$ and the three inequalities 
(a) $\Delta(a) \leqq k \lim _{r=1} \sup \frac{P(r, a, \sigma(r))}{T(r)}+I^{*}(\sigma(r), f)$,

(b) $\delta(a) \leqq k \lim \sup _{r=1} \frac{P(r, a, \sigma(r))}{T(r)}+I_{*}(\sigma(r), f)$,

(c) $\delta(a) \leqq k \liminf _{r=1} \frac{P(r, a, \sigma(r))}{T(r)}+I^{*}(\sigma(r), f)$

are satisfied, or

(ii) $r=1$ is a limit point of both $\&(a, \sigma(r))$ and $C \&(a, \sigma(r))$ and the inequality

$$
\begin{aligned}
\delta(a) & \leqq k \liminf _{r^{\prime}=1} \frac{P\left(r^{\prime}, a, \sigma\left(r^{\prime}\right)\right)}{T\left(r^{\prime}\right)}+I^{*}(\sigma(r), f) \quad\left[r^{\prime} \in £(a, \sigma(r))\right] \\
& \leqq k \lim _{r=1} \sup \frac{P(r, a, \sigma(r))}{T(r)}+I^{*}(\sigma(r), f)
\end{aligned}
$$

is satisfied, or

(iii) $r=1$ is not a limit point of $\&(a, \sigma(r))$, where in general $k=\pi$, but under condition $(*) k=\pi / 2$.

The method of proof is that of Theorem III. (21.1)(a) and (b) are deduced from Corollary II. 2.2. To prove (21.1) (a) we may assume $0 \leqq \mathrm{I}^{*}(\sigma(r), f)<\Delta(a)$ and, as in $\$ 17$, put

$$
k \lim _{r=1} \frac{p(r)}{T(r)}=\Delta(a)-I^{*}(\sigma(r), f)-\epsilon,
$$

where $\epsilon>0$ is arbitrarily small. Then, by (20.3)(a) and (20.4)(a), $r=1$ is a limit point of $C E(a, \sigma(r), p(r))$. By hypothesis $r=1$ is not a limit point of $C \&(a, \sigma(r))$ and it follows that, for $r^{\prime \prime} \in C E(a, \sigma(r), p(r))$ and sufficiently near to $r=1$,

$$
P\left(r^{\prime \prime}, a, \sigma\left(r^{\prime \prime}\right)\right)>p\left(r^{\prime \prime}\right) .
$$

For otherwise there must be at least one unbounded domain $G\left(r^{\prime \prime}, a, \sigma\left(r^{\prime \prime}\right)\right)$. Hence

$$
\begin{aligned}
k \lim \sup \frac{P(r, a, \sigma(r))}{r=1} & \geqq k \lim _{r^{\prime \prime}=1} \frac{P\left(r^{\prime \prime}, a, \sigma\left(r^{\prime \prime}\right)\right)}{T\left(r^{\prime \prime}\right)} \\
& \geqq k \lim _{r=1} \frac{p(r)}{T(r)}=\Delta(a)-I^{*}(\sigma(r), f)-\epsilon
\end{aligned}
$$

and, since $\epsilon>0$ is arbitrary, (21.1)(a) follows.

To prove (21.1)(b) we merely repeat the argument with 


$$
0 \leqq I_{*}(\sigma(r), f)<\delta(a)
$$

and

$$
k \lim _{r=1} \frac{p(r)}{T(r)}=\delta(a)-I_{*}(\sigma(r), f)-\epsilon .
$$

To prove (21.1)(c) we use Corollary II. 2.4. Assume

$$
0 \leqq I^{*}(\sigma(r), f)<\delta(a)
$$

and put

$$
k \lim _{r=1} \frac{p(r)}{T(r)}=\delta(a)-I^{*}(\sigma(r), f)-\epsilon .
$$

Then, for all sufficiently large $r<1$, it follows from (20.7) and (20.8) that $P(r, a, \sigma(r))>p(r)$ since by hypothesis $r \in \&(a, \sigma(r))$. Hence

$$
k \liminf _{r=1} \frac{P(r, a, \sigma(r))}{T(r)} \geqq k \lim _{r=1} \frac{p(r)}{T(r)}=\delta(a)-I^{*}(\sigma(r), f)-\epsilon
$$

and this proves $(21.1)(\mathrm{c})$.

To prove (ii) we observe that in the product set $\&(a, \sigma(r)) \times C E(a, \sigma(r)$, $p(r))$ we have $P(r, a, \sigma(r))>p(r)$ and that under the condition (21.2), $r=1$ is not a limit point of $E(a, \sigma(r), p(r))$. Therefore all the points of $\&(a, \sigma(r))$ sufficiently near to $r=1$ are points of the product set.

22. Deductions from Theorem IV. The formal pattern is that of $\$ 18$. First, if for a $\sigma(r)$ of index zero $r=1$ is not a limit point of $C \&(a, \sigma(r))$ and if $P(r, a, \sigma(r))=o(T(r))$, then $\Delta(a)=0$; while if

$$
\liminf _{r=1} \frac{P(r, a, \sigma(r))}{T(r)}=0,
$$

then $\delta(a)=0$. This generalizes Corollaries A.1 and A.3. Proceeding further, the following two corollaries, which are immediate deductions from Theorem IV, are the hyperbolic analogues of Corollaries III.1 and III.2.

Corollary IV.1. Suppose that $f(z)$ is meromorphic in $|z|<1$ and that $\Delta(a)>0$. Then if for a given $\sigma(r)$

$$
k \lim \sup \frac{P(r, a, \sigma(r))}{T(r)} \leqq \eta \Delta(a), \quad 0 \leqq \eta<1,
$$

and $r=1$ is not a limit point of $C \&(a, \sigma(r))$, we have

$$
I^{*}(\sigma(r), f) \geqq(1-\eta) \Delta(a) .
$$

Similarly, if $\delta(a)>0$ and if 


$$
k \limsup _{r=1} \frac{P(r, a, \sigma(r))}{T(r)} \leqq \eta \delta(a)
$$

and $r=1$ is not a limit point of $C \mathscr{E}(a, \sigma(r))$, we have $I^{*}(\sigma(r), f) \geqq(1-\eta) \delta(a)$; or under the weaker condition that $r=1$ is a limit point of $\&(a, \sigma(r))$ we have

$$
I^{*}(\sigma(r), f) \geqq(1-\eta) \delta(a) .
$$

Corollary IV.2. Suppose that $f(z)$ is meromorphic in $|z|<1$ and that $\Delta(a)>0$. Then for $a \sigma(r)$ such that

$$
I^{*}(\sigma(r), f) \leqq \theta \Delta(a),
$$$$
0 \leqq \theta<1
$$

and $r=1$ is not a limit point of $C \&(a, \sigma(r))$, we have

$$
k \lim \sup \frac{P(r, a, \sigma(r))}{T(r)} \geqq(1-\theta) \Delta(a) .
$$

Similarly, if $\delta(a)>0$ and $\sigma(r)$ is such that

$$
I^{*}(\sigma(r), f) \leqq 6 \delta(a)
$$

and $r=1$ is not a limit point of $C \&(a, \sigma(r))$, we have

$$
k \liminf _{r=1} \frac{P(r, a, \sigma(r))}{T(r)} \geqq(1-\theta) \delta(a) ;
$$

or under the weaker condition that $r=1$ is a limit point of $\mathbb{E}(a, \sigma(r))$ we have

$$
k \lim \sup \frac{P(r, a, \sigma(r))}{T(r)} \geqq k \liminf _{r^{\prime}=1} \frac{P\left(r^{\prime}, a, \sigma\left(r^{\prime}\right)\right)}{T\left(r^{\prime}\right)} \geqq(1-\theta) \delta(a),
$$

where $r^{\prime} \in(\$(a, \sigma(r))$.

In both of these corollaries in general $k=\pi$, but $k=\pi / 2$ if condition (*) is satisfied.

Corollary IV.2 stands in the same relation to Theorem $\mathrm{B}^{\prime}$, which it contains as a special case, as does Corollary III.2. to Theorem C.

The condition that $r=1$ is not a limit point of $C \&(a, \sigma(r))$ is satisfied in known examples. A case in point has been discussed by Valiron [2], pursuing an idea of Fatou. Koenigs' function $K(z)$ is regular and of finite order in $|z|<1$ and, given an arbitrarily large $\sigma$, all the $G(1 / K, \sigma)$ are bounded so that $C \&(0, \sigma)=C \&(1 / K, \sigma)$ is void. It follows that for any nonincreasing $\sigma(r)$ and any finite $a$ the set $C \&(a, \sigma(r))$ is void. It can also be shown that $P(r, 1 / K, \sigma) \leqq 1$ for $r(\sigma)<r<1$ from which it follows that $\Delta(a)=0$ for all finite $a$. Analogous examples have been discussed by Lusin and Privaloff [1]. None of these examples, however, enables us to test the exactness of the inequalities (21.1).

An example of the opposite kind where the condition that $r=1$ is not a 
limit point of $\mathbb{E}(a, \sigma(r))$ is evidently furnished by any function having $a$ as an asymptotic value provided that $\sigma(r)$ is not a function that tends too rapidly to zero. Thus $\infty$ is an asymptotic value of $K(z)$ and $r=1$ is not a limit point of $\mathbb{E}(\infty, \sigma)=\mathscr{E}(K, \sigma)$ for any fixed $\sigma$; and this remains true if $\sigma$ is replaced by a $\sigma(r) \rightarrow 0$ sufficiently slowly as $r \rightarrow 1$. Another obvious example is furnished by the modular function which has one of the radial limits 0,1 , or $\infty$ at each one of the enumerable set of vertices of the modular figure. For the condition to be satisfied for a $\sigma(r) \rightarrow 0$ it is not, of course, necessary that $a$ should be an asymptotic value; it is plainly sufficient for $a$ to be a limit of asymptotic values, and again we can make no assertion as to necessity.

\section{Proof of Theorem II}

23. Two lemmas of Selberg. The proof of Theorem II turns upon two important lemmas recently proved by Selberg. The first of these is:

LEMMA 1(22). Let $G$ be a finite schlicht domain in the z-plane bounded by a finite number of analytic arcs and let $Q$ be the open set of points of $|z|=r$ which are internal points of $G$. Suppose further that $G$ is such that $Q$ consists of a finite number of distinct arcs whose total length is $\theta r . z_{0}$ is an internal point of $G$ and $g\left(z, G, z_{0}\right)$ is the Green's function for $G$ having its pole at $z_{0}$. Then

$$
\int_{Q} g\left(r e^{i \phi}, G, z_{0}\right) d \phi \leqq \pi^{2} \tan \frac{\theta}{4} .
$$

We also require the following additional inequality. Suppose that $z=0$ is neither an internal point nor a frontier point of $G$ and let $r_{1}$ be the shortest distance from $z=0$ to the frontier of $G$. Then $G$ is contained in the domain outside the circle $|z|=r_{1}$ so that

$$
g\left(z, G, z_{0}\right) \leqq \log \left|\frac{r_{1}^{2}-z \bar{z}_{0}}{r_{1}\left(z-z_{0}\right)}\right| .
$$

For the right-hand member is the Green's function for the domain outside $|z|=r_{1}$ and having $z_{0}$ as pole. Now

$$
\int_{0}^{2 \pi} \log \left|\frac{r_{1}^{2}-r e^{i \phi} \bar{z}_{0}}{r_{1}\left(r e^{i \phi}-z_{0}\right)}\right| d \phi \leqq 2 \pi \log ^{+} \frac{r}{r_{1}}
$$

and so

$$
\int_{Q} g\left(r e^{i \phi}, G, z_{0}\right) d \phi \leqq 2 \pi \log ^{+} \frac{r}{r_{1}}
$$

which is the inequality required.

(22) Selberg [5, p. 311, Satz 1]. 
Now instead of the circumference $|z|=r$ consider a straight line intersecting $G$. Without loss of generality we may assume this line to be the real axis. Let the finite set $H$ of its segments interior to $G$ be of total length $L$. The second of Selberg's lemmas that we require is:

Lemma 2(23). $G$ is a schlicht domain in the plane of $z=x+i y$ bounded by a finite number of analytic arcs, $z_{0}$ is an internal point of $G$ and $g\left(z, G, z_{0}\right)$ the corresponding Green's function with its pole at $z_{0}$. If the set $H$ of segments of the axis of $x$ contained in $G$ is of total length $L$, then

$$
\int_{H} g\left(z, G, z_{0}\right) d x \leqq \frac{\pi}{2} L
$$

This result sharpens and generalizes that proved by Teichmüller under the restriction that $G$ is simply-connected and giving a less favorable coefficient of $L$.

24. Proof of inequalities (15.1) and (15.2). We are now in a position to prove Theorem II. The proof falls into two parts. In this section we prove (15.1) and (15.2) by Selberg's method, using Lemma 1 and (23.2); and in $\$ 25$ we proved (15.3) by Teichmüller's method, using Lemma 2.

In proving Theorem II there is evidently no restriction in taking $a=\infty$, for we have only to make the transformation $w_{1}=1 /(w-a)$ and observe that

$$
\begin{gathered}
m\left(r, w_{1}\right)=m(r, a), \\
N\left(r, w_{1}\right)=N(r, a), \\
T\left(r, w_{1}\right)=T(r, w)+O(1) .
\end{gathered}
$$

Our hypothesis then is that $R \leqq \infty$ is a limit point of the set $E=E(\infty, \sigma(r)$, $p(r)$ ) where $\sigma(r)$ and $p(r)$ satisfy the conditions of the theorem. For a given $r \in E$ consider the domains $G_{\nu}(r)=G_{\nu}(r, \infty, \sigma(r)), \nu \leqq \lambda(r)$. By hypothesis, each of the domains $G_{\nu}(r)$ is "bounded" and contains at most $p(r)$ poles of $f(z)$, while on the boundary of $G_{\nu}(r)$

$$
|f(z)|=1 / \sigma(r) .
$$

Hence in each of the domains $G_{\nu}(r)$, where $\nu \leqq \lambda(r), \log |\sigma(r) f(z)|$ can be represented as the sum of not more than $p(r)$ Green's functions for $G_{\nu}(r)$ having the poles of $f(z)$ in this domain as poles of the Green's functions. Now denote by $Q_{\nu}$ the arcs of $|z|=r$ interior to $G_{\nu}(r)$ and let their total length be $\theta_{\nu} r$. Then we may apply Lèmma 1 to $G_{\nu}(r)$ and obtain

$$
\int_{Q_{\nu}} \log \left|\sigma(r) f\left(r e^{i \phi}\right)\right| d \phi \leqq p(r) \pi^{2} \tan \frac{\theta_{\nu}}{4}
$$

(29) Selberg [6, p. 330]. 
by $(23.1)$.

We may evidently put $\theta_{1} \geqq \theta_{2} \geqq \cdots \geqq \theta_{\lambda(r)}$ and, since the $Q_{\nu}$ do not overlap, it follows that $\theta_{\nu} \leqq \pi$ for $\nu=2,3, \cdots, \lambda(r)$ and $\sum_{\nu=2}^{\lambda(r)} \theta_{\nu}<2 \pi$. Hence

$$
\sum_{\nu=2}^{\lambda(r)} \int_{Q_{\nu}} \log \left|\sigma(r) f\left(r e^{i \phi}\right)\right| d \phi \leqq p(r) \pi^{2} \sum_{\nu=2}^{\lambda(r)} \tan \frac{\theta_{\nu}}{4} \leqq p(r) \pi \sum_{\nu=2}^{\lambda(r)} \theta_{\nu}<2 \pi^{2} p(r) .
$$

We now have to deal with $G_{1}(r)$. For this we rely upon (23.2). Take first the case $R=\infty$. Then there is an $r_{2}$ such that for all $r \in E$ and greater than $r_{2}$ the domain $G_{1}(r, \infty, \sigma(r))$ lies outside the circle $|z|=1$. For, given $r_{1} \geqq 1$ and belonging to $E$, the $G_{\mu}\left(r, \infty, \sigma\left(r_{1}\right)\right)$, where $\mu \leqq \lambda\left(r_{1}\right)$, are bounded and therefore contained in a circle $|z|<r_{2}$. Now, for $r_{2}<r \in E, \sigma(r) \leqq \sigma\left(r_{1}\right)$ and so the $G_{\nu}(r, \infty, \sigma(r))$, where $\nu \leqq \lambda(r)$, are contained in domains $G\left(r, \infty, \sigma\left(r_{1}\right)\right)$ which may or may not be bounded but none of which intercepts $|z|=r_{1}$ since none of them can be a $G_{\mu}\left(r_{1}, \infty, \sigma\left(r_{1}\right)\right)$. Hence all the $G_{\nu}(r, \infty, \sigma(r)), \nu=1,2, \cdots$, $\lambda(r)$, lie outside $|z|=r_{1} \geqq 1$ and so, applying (23.2) to $G_{1}(r, \infty, \sigma(r))$, we have

$$
\int_{Q_{1}} \log \left|\sigma(r) f\left(r e^{i \phi}\right)\right| d \phi \leqq 2 \pi p(r) \log \frac{r}{r_{1}} \leqq 2 \pi p(r) \log r .
$$

Now by (24.2) and (24.3)

$$
\begin{aligned}
m(r, \infty) & \leqq \frac{1}{2 \pi} \int_{0}^{2 \pi} \log ^{+}\left|\sigma(r) f\left(r e^{i \phi}\right)\right| d \phi+\log ^{+} \frac{1}{\sigma(r)} \\
& =\frac{1}{2 \pi} \sum_{\nu=1}^{\lambda(r)} \int_{Q_{\nu}} \log \left|\sigma(r) f\left(r e^{i \phi}\right)\right| d \phi+\log ^{+} \frac{1}{\sigma(r)} \\
& \leqq(\pi+\log r) p(r)+\log ^{+} \frac{1}{\sigma(r)} .
\end{aligned}
$$

Hence for any value $a$ and $r \in E(a, \sigma(r), p(r))$ satisfying $r>r_{2}$

$$
m(r, a)<(\pi+\log r) p(r)+\log ^{+}(1 / \sigma(r))
$$

and, since $m(r, a)=O(1)$ for $r \leqq r_{2},(15.1)$ is proved.

In the case $R<\infty$ the argument is similar. Given an arbitrarily small positive $\epsilon$ we choose $r_{1}=r_{1}(\epsilon) \in E$ satisfying $R /(1+\epsilon) \leqq r_{1}<R$. By hypothesis the domains $G_{\mu}\left(r_{1}, \infty, \sigma\left(r_{1}\right)\right)$, where $\mu \leqq \lambda\left(r_{1}\right)$, are contained in $|z|<r_{2}<R$. Now, for $r_{2}<r<R, r \in E$, we have $\sigma(r) \leqq \sigma\left(r_{1}\right)$ and so the $G_{\nu}(\sigma, \infty, \sigma(r))$, $\nu \leqq \lambda(r)$, are contained in domains $G\left(r, \infty, \sigma\left(r_{1}\right)\right)$, none of which intercepts $|z|=r_{1}$. Hence

(24.5) $\quad \int_{Q_{1}} \log \left|\sigma(r) f\left(r e^{i \phi}\right)\right| d \phi \leqq 2 \pi p(r) \log \frac{r}{r_{1}}<2 \pi p(r) \log \frac{R}{r_{1}}<2 \pi \epsilon p(r)$.

Combining (24.5) with (24.2) we have 


$$
m(r, \infty)<(\pi+\epsilon) p(r)+\log ^{+}(1 / \sigma(r)) .
$$

The same inequality holds for $m(r, a)$ and, since $\epsilon$ is arbitrary and $m(r, a)$ $=O(1)$ for $r \leqq r_{2},(15.2)$ is proved.

25. Proof of (15.3). As stated in $\$ 15$ we shall prove that (15.3) is satisfied for $R \leqq \infty$ and all $r \in E$ under condition $\left(^{* *}\right)$. Theorem II then follows a fortiori.

Suppose then that condition $\left({ }^{* *}\right)$ is satisfied for $a=\infty$. As we saw in $\$ 24$, given $r_{1}<R$, we can find $r_{2}$, where $r_{1}<r_{2}<R$, such that for $r_{2}<r \in E$ the domains $G_{\nu}(r)=G_{\nu}(r, \infty, \sigma(r))$ all lie outside $|z|=r_{1}$. We may choose $r_{1}>B$ and $r_{2}>A$. Then all the $G_{\nu}(r)$, if any exist for this value of $r$, lie outside $|z|=B$ and none of them contains a closed curve enclosing this circumference. Hence none of them contains a closed curve enclosing the origin $z=0$ which therefore lies in an "unbounded" domain $D_{r}$ excluded from all the $G_{\nu}(r)$. The origin can therefore be joined to a point of $|z|=R$ or to $z=\infty$, as the case may be, by a continuous curve $\gamma$ contained in $D_{r}$. Moreover, the domains $G_{\nu}(r)$ all lie outside the circle $|z|=r_{1}$ and inside a circle $|z|=r^{\prime}<R \leqq \infty$ and we may certainly assume that $\gamma$ has only one point on each of these circles. Denote by $\gamma\left(r_{1}, r^{\prime}\right)$ the arc of $\gamma$ in the annulus $r_{1}<|z|<r^{\prime}$ which, as we have seen, contains the domains $G_{\nu}(r)$.

The transformation

$$
\zeta=\log z=\log r+i \phi
$$

maps the annulus $r_{1}<|z|<r^{\prime}$, cut along $\gamma\left(r_{1}, r^{\prime}\right)$, upon a strip-like domain $\Xi\left(r_{1}, r^{\prime}\right)$ bounded by two transforms of $\gamma\left(r_{1}, r^{\prime}\right)$, corresponding points of which are separated by a distance $2 \pi$, and by segments of $\Re \zeta=\log r$ and $\Re \zeta=\log r^{\prime}$ each of length $2 \pi$. Every $G_{\nu}(r)$ is transformed into a finite closed domain $F_{\nu}(\log r)$ contained in $\Xi^{\prime}\left(r_{1}, r^{\prime}\right)$, and the circumference $|z|=r$ which intercepts all the $G_{\nu}(r)$ is transformed into a cross-cut of $\Xi\left(r_{1}, r^{\prime}\right)$ parallel to the $\phi$-axis and of length $2 \pi$. The arcs $Q_{\nu}$ transform into nonoverlapping segments of this cross-cut whose total length we denote by $L_{\nu}$. Plainly $\sum_{v=1}^{\lambda(r)} L<2 \pi$.

The Green's function $g\left(z, G_{\eta}, z_{m}\right)$ transforms into $g\left(\zeta, F_{\nu}, \zeta_{m}\right)$, where $z_{m}$ denotes a pole of $f(z)$ in $G_{\nu}(r)$ and $\zeta_{m}$ is its transform in $F_{\nu}(\log r)$.

As before, for $z$ in $G_{v}(r)$ we can express $\log |\sigma(r) f(z)|$ as a sum of Green's functions and we have

$$
\begin{aligned}
\int_{0}^{2 \pi} \log ^{+}\left|\sigma(r) f\left(r e^{i \phi}\right)\right| d \phi & =\sum_{\nu=1}^{\lambda(r)} \int_{Q_{\nu}} \log \left|\sigma(r) f\left(r e^{i \phi}\right)\right| d \phi \\
& =\sum_{\nu=1}^{\lambda(r)} \sum_{m} \int_{Q_{\nu}} g\left(\zeta, F_{\nu}, \zeta_{m}\right) d \phi \\
& \leqq \frac{\pi}{2} p(r) \sum_{\nu=1}^{\lambda(r)} L_{\nu}<\pi^{2} p(r)
\end{aligned}
$$


by Lemma 2 since the number of poles in each $G_{v}(r)$ does not exceed $p(r)$. Hence

$$
m(r, \infty)<\frac{\pi}{2} p(r)+\log ^{+} \frac{1}{\sigma(r)}
$$

provided that $A<r_{2}<r \in E$. The same inequality holds for $m(r, a)$ with $A<r_{2}$ $<r \in E(a, \sigma(r), p(r))$ and, since $m(r, a)=O(1)$ for $r \leqq r_{2}$, (15.3) is proved. This completes the proof of Theorem II.

\section{BIBLIOGRAPHY}

Сн. BLanc

1. Les surfaces de Riemann des fonctions meromorphes, Comment. Math. Helv. vol. 9 (1937). H. Cartan

1. Sur les valeurs exceptionelles des fonctions méromorphes dans tout le plan, C. R. Acad. Sci. Paris vol. 190.

E. F. Collingwood

1. Sur les valeurs exceptionelles des fonctions entières d'ordre fini, C. R. Acad. Sci. Paris vol. 179 (1924).

2. On meromorphic and integral functions, J. London Math. Soc. vol. 5 (1930).

3. Sur certains ensembles définis pour les fonctions meromorphes, C. R. Acad. Sci. Paris vol. 227 (1948) pp. 615-617, 1119-1120.

4. Une inégalitê dans la thêorie des fonctions méromorphes, C. R. Acad. Sci. Paris vol. 227 (1948) pp. 709-711, 1119-1120.

5. Inégalités relatives à la distribution des valeurs d'une fonction méromorphe dans le plan fini. C. R. Acad. Sci. Paris vol. 227 (1948) pp. 749-751, 1119-1120.

6. Inégalités relatives da la distribution des valeurs d'une fonction méromorphe dans le cercle unite, C. R. Acad. Sci. Paris vol. 227 (1948) pp. 813-815, 1119-1120.

A. Dinghas

1. Über Ausnahmegebiete meromorpher Funktionen, Math. Zeit. vol. 45 (1939).

W. GRoss

1. Über die Singularitäten analytischer Funktionen, Monatshefte für Mathematik und Physik vol. 29 (1918).

2. Zum Verhalten analytischer Funktionen in der Umgebung singularer Stellen, Math. Zeit. vol. 2 (1918).

3. Eine ganze Funktion, fitr die jede komplexe Zahl Konvergenzwert ist, Math. Ann. vol. 79 (1918).

F. IVERSEN

1. Rercherches sur les fonctions inverses des fonctions méromorphes, Thèse, Helsingfors, (1914).

2. Sur une fonction entière dont la fonction inverse presente un ensemble de singularites transcendantes de la puissance du continu, Ofversigt af Finska Vetenskaps-societetens Förhandlingar vol. 43 Afdelning A No. 3 (1915-1916).

3. Sur quelques propriettes des fonctions monogènes au voisinage d'un point singulier, ibid. No. 4 (1915-1916).

4. Sur quelques fonctions entières qui admettent des valeurs asymptotiques finis, Öfversigt af Finska Vetenskaps-societetens Förhandlingar vol. 41 No. 1 (1918).

5. Zum Verhalten analytischer Funktionen in Bereichen, daren Rand eine wesentliche Singularität enthält, Öfversigt af Finska Vetenskaps-societetens Förhandlingar vol. 44 Afdelning A No. 4 (1921). 


\section{S. KAKUTANI}

1. On the exceptional value of meromorphic functions, Proceedings of the Physico-Mathematical Society of Japan (3) vol. 17 (1935).

N. Lusin and J. Privaloff

1. Sur l'unicité et la multiplicitê des fonctions analytiques, Ann. Ecole Norm. (3) vol. 42 (1925) pp. 143-191.

A. J. MACINTYRE

1. A theorem concerning meromorphic functions of finite order, Proc. London Math. Soc. vol. 39 (1935).

P. MONTEL

1. Lȩ̧ons sur les fonctions univalentes ou multivalentes, Collection Borel, Gauthier-Villars, 1933.

M. PARREAU

1. Sur le thêoreme de Collingwood-Cartan, C. R. Acad. Sci. Paris vol. 227 (1948) pp. 13231325.

A. Pfluger

1. Zur Defektrelation ganzer Funktionen endlicher Ordnung, Comment. Math. Helv. vol. 19 (1946).

MME. LAURENT-SCHWARTZ

1. Sur une propriété de la fonction $M(r, A)$ de $M$. Nevanlinna, C. R. Acad. Sci. Paris vol. 210 (1940).

2. Exemple d'une fonction méromorphe ayant des valeurs deficientes non asymptotiques, $\mathrm{C} . \mathrm{R}$. Acad. Sci. Paris vol. 212 (1941)

H. L. SELBERG

1. Beiträge zur Theorie der Algebroiden Funktionen, Avhandlinger Norske VidenskapsAkademi, Oslo, Matematisk-Naturvidenskapelig Klasse (1931).

2. Algebroide und Umkehrfunktionen Abelscher Integrale, ibid (1934).

3. Über einen Satz von Collingwood, Archiv för Mathematik og Naturvidenskab (1944).

4. Eine Ungleichung der Potentialtheorie und ihre Anwendung in der Theorie der meromorphen Funktionen, Comment. Math. Helv. vol. 18 (1946) pp. 309-326.

4. Eine Ungleichung der Potentialtheorie und ihre Anwendung in der Theorie der meromorphen Funktionen, Comment. Math. Helv. vol. 18 (1946) pp. 309-326.

5. Über eine Ungleichung der Potentialtheorie, ibid. pp. 327-330.

O. TEICHMÜLLER

1. Eine Umkehrung des zweiten Hauptsatzes der Wertverteilungslehre, Deutsche Mathematik vol. 2 (1937) pp. 97-107.

2. Vermutungen und Sätze ïber die Wertverteilung gebrochener Functionen endlicher Ordnung, Deutsche Mathematik vol. 4 (1939) pp. 163-190.

3. Einfache Beispiele zur Wertverteilungslehre, Deutsche Mathematik vol. 7 (1942-1944) pp. 360-368.

Y. TUMURA

1. Sur quelques propriêtés d'une classe des fonctions meromorphes, Proceedings of the E. UlLRICH Physico-Mathematical Society of Japan. (3) vol. 18.

1. Uber eine Anwendung des Verzerrungssatzes auf meromorphe Funktionen, J. Reine Angew. Math. vol. 166 (1932) pp. 220-234.

2. Review of Selberg [2], Zentralblatt für Mathematik und ihre Grenzgebiete vol. 10 (1935).

3. Flächenbau und Wachstumsorḋnung bei gebrochener Funktionen, J. Deutschen Math. Verein. vol. 46 (1936) pp. 232-274.

4. Flächenbau und Wertverteilung, Neuvième Congres des Mathématiciens Scandinaves, Helsingfors, 1939, pp. 179-200. 
G. VALIRON

1. Sur la distribution des valeurs des fonctions méromorphes, Acta Math. vol. 47 (1925).

2. Sur les singularités de certaines fonctions holomorphes et de leurs inverses, Journal de Mathematiques (3) vol. 15 (1936).

K. Yosida

1. A theorem concerning the derivatives of meromorphic functions, Proceedings of the PhysicoMathematical Society of Japan (3) vol. 17 (1935).

LILBURN TOWER,

Alnwick, England. 